\title{
Arithmetic duality theorems for 1-motives
}

\author{
David Harari and Tamás Szamuely
}

\section{Introduction}

Duality theorems for the Galois cohomology of commutative group schemes over local and global fields are among the most fundamental results in arithmetic. Let us briefly and informally recall some of the most famous ones.

Perhaps the earliest such result is the following. Given an algebraic torus $T$ with character group $Y^{*}$ defined over a $p$-adic field $K$, cup-products together with the isomorphism $\operatorname{Br}(K)=H^{2}\left(K, \mathbf{G}_{m}\right) \cong \mathbf{Q} / \mathbf{Z}$ given by the invariant map of the Brauer group of $K$ define canonical pairings

$$
H^{i}(K, T) \times H^{2-i}\left(K, Y^{*}\right) \rightarrow \mathbf{Q} / \mathbf{Z}
$$

for $i=0,1,2$. The Tate-Nakayama duality theorem (whose original form can be found in [27]) then asserts that these pairings become perfect if in the cases $i \neq 1$ we replace the groups $H^{0}$ by their profinite completions. Note that this theorem subsumes the reciprocity isomorphism of local class field theory which is the case $i=0, T=\mathbf{G}_{m}$.

Next, in his influential exposé [28], Tate observed that given an abelian variety $A$, the Poincaré pairing between $A$ and its dual $A^{*}$ enables one to construct similar pairings

$$
H^{i}(K, A) \times H^{1-i}\left(K, A^{*}\right) \rightarrow \mathbf{Q} / \mathbf{Z}
$$

for $i=0,1$ and he proved that these pairings are also perfect.

The last result we recall is also due to Tate. Consider now an abelian variety $A$ over a number field $k$, and denote by $\amalg^{1}(A)$ the Tate-Shafarevich group formed by isomorphism classes of torsors under $A$ that split over each completion of $k$. Then Tate constructed a duality pairing

$$
\amalg^{1}(A) \times \amalg^{1}\left(A^{*}\right) \rightarrow \mathbf{Q} / \mathbf{Z}
$$

(generalising earlier work of Cassels on elliptic curves) and announced in [29] that this pairing is nondegenerate modulo divisible subgroups or else, if 
one believes the widely known conjecture on the finiteness of $\amalg^{1}(A)$, it is a perfect pairing of finite abelian groups. Similar results for tori are attributed to Kottwitz in the literature; indeed, the references [10] and 11] contain such statements, but without (complete) proofs.

In this paper we establish common generalisations of the results mentioned above for 1-motives. Recall that according to Deligne, a 1-motive over a field $F$ is a two-term complex $M$ of $F$-group schemes $[Y \rightarrow G]$ (placed in degrees -1 and 0 ), where $Y$ is the $F$-group scheme associated to a finitely generated free abelian group equipped with a continuous $\mathrm{Gal}(F)$-action and $G$ is a semi-abelian variety over $F$, i.e. an extension of an abelian variety $A$ by a torus $T$. As we shall recall in the next section, every 1-motive $M$ as above has a Cartier dual $M^{*}=\left[Y^{*} \rightarrow G^{*}\right]$ equipped with a canonical (derived) pairing $M \otimes^{\mathbf{L}} M^{*} \rightarrow \mathbf{G}_{m}$ [1] generalising the ones used above in the cases $M=[0 \rightarrow T]$ and $M=[0 \rightarrow A]$. This enables one to construct duality pairings for the Galois hypercohomology groups of $M$ and $M^{*}$ over local and global fields.

Let us now state our main results. In Section 2, we shall prove:

Theorem 0.1 Let $K$ be a local field and let $M=[Y \rightarrow G]$ be a 1-motive over $K$. For $i=-1,0,1,2$ there are canonical pairings

$$
\mathbf{H}^{i}(K, M) \times \mathbf{H}^{1-i}\left(K, M^{*}\right) \rightarrow \mathbf{Q} / \mathbf{Z}
$$

inducing perfect pairings between

1. the profinite group $\mathbf{H}_{\wedge}^{-1}(K, M)$ and the discrete group $\mathbf{H}^{2}\left(K, M^{*}\right)$;

2. the profinite group $\mathbf{H}^{0}(K, M)^{\wedge}$ and the discrete group $\mathbf{H}^{1}\left(K, M^{*}\right)$.

Here the groups $\mathbf{H}^{0}(K, M)^{\wedge}$ and $\mathbf{H}_{\wedge}^{-1}(K, M)$ are obtained from the corresponding hypercohomology groups by certain completion procedures explained in Section 2. We shall also prove there a generalisation of the above theorem to 1-motives over henselian local fields of mixed characteristic and show that in the duality pairing the unramified parts of the cohomology are exact annihilators of each other.

Now let $M$ be a 1-motive over a number field $k$. For all $i \geq 0$ define the Tate-Shafarevich groups

$$
\amalg^{i}(M)=\operatorname{Ker}\left[\mathbf{H}^{i}(k, M) \rightarrow \prod_{v} \mathbf{H}^{i}\left(\hat{k}_{v}, M\right)\right]
$$

where the product is taken over completions of $k$ at all (finite and infinite) places of $k$. Our main result can then be summarised as follows. 
Theorem 0.2 Let $k$ be a number field and $M$ a 1-motive over $k$. There exist canonical pairings

$$
\amalg^{i}(M) \times \amalg^{2-i}\left(M^{*}\right) \rightarrow \mathbf{Q} / \mathbf{Z}
$$

for $i=0,1$ which are non-degenerate modulo divisible subgroups.

In fact, here for $i=0$ the group $\amalg^{0}(M)$ is finite, so the left kernel is trivial. Assuming the finiteness of the (usual) Tate-Shafarevich group of an abelian variety one derives that for $i=1$ the pairing is a perfect pairing of finite groups.

The pairings used here can be defined purely in terms of Galois cohomology (see Section 6); however, to prove the duality isomorphisms we first construct pairings using étale cohomology in Sections 3 and 4, and then in the last section we compare them to the Galois-cohomological one which in the case of abelian varieties gives back the classical construction of Tate.

Finally, in Section 5 we establish a twelve-term Poitou-Tate type exact sequence similar to the one for finite modules, assuming the finiteness of the Tate-Shafarevich group. The reader is invited to look up the precise statement there.

Since this is a paper containing the word "motive", it is appropriate to explain our motivations for establishing the generalisations offered here. The first of these should be clear from the above: working in the context of 1-motives gives a unified and symmetric point of view on the classical duality theorems cited above and gives more complete results than those known before. As an example, we may cite the duality between $\amalg^{i}(T)$ and $\amalg^{3-i}\left(Y^{*}\right)(i=1,2)$ for an algebraic torus $T$ with character group $Y^{*}$ which is a special case of Theorem 0.2 above (see Section 4 ); it is puzzling to note that the reference [18] only contains the case $i=1$, whereas [16] only the case $i=2$. Another obvious reason is that due to recent spectacular progress in the theory of mixed motives there has been a regain of interest in 1-motives as well; indeed, the category of 1-motives over a field (with obvious morphisms) is equivalent, up to torsion, to the subcategory of the triangulated category of mixed motives (as defined, e.g., by Voevodsky) generated by motives of varieties of dimension at most 1 .

But there is a motivation coming solely from the arithmetic duality theory. In fact, if one tries to generalise the classical duality theorems of Tate to a semi-abelian variety $G$, one is already confronted to the fact that the only reasonable definition for the dual of $G$ is the dual $\left[Y^{*} \rightarrow A^{*}\right]$ of the 1-motive $[0 \rightarrow G]$, where actually $A^{*}$ is the dual of the abelian quotient of $G$ and $Y^{*}$ is the character group of its toric part. Duality results of this type are needed for the study of the arithmetic of $G$ over local and global fields: for instance, such a question (which we shall address elsewhere) is the unicity of 
the Brauer-Manin obstruction to the Hasse principle for rational points on torsors under $G$. (This question was raised by Skorobogatov in [26], p. 133).

In conclusion to this introduction, we cannot resist the temptation of recalling the basic example of a dual pair of 1-motives "appearing in nature". For this, let $X$ be a smooth projective variety over an algebraically closed field. It is well-known that the Albanese variety $\mathrm{Alb}_{X}$ and the Picard variety $\operatorname{Pic}_{X}^{0}$ of $X$ are dual abelian varieties. Now consider an open subvariety $U \subset X$. For such a $U$ a natural object to consider is the generalised Albanese variety $\widehat{\mathrm{Alb}}_{U}$ defined by Serre 24]: it is a semi-abelian variety equipped with a morphism $U \rightarrow \widehat{\operatorname{Alb}}_{U}$ universal for morphisms of $U$ into semi-abelian varieties that send a fixed base point to 0. According to [25] (see also [21]) the dual of the 1-motive $\left[0 \rightarrow \widehat{\operatorname{Alb}}_{U}\right]$ is the 1-motive $\left[Y \rightarrow \operatorname{Pic}_{X}^{0}\right]$, where $Y$ is the group of divisors on $X$ supported in $X \backslash U$ and algebraically equivalent to 0 , and the map is the divisor class map.

After the first version of this paper has been posted on the Web, N. Ramachandran has kindly drawn our attention to the paper of Berkovich [2] where duality theorems for the Galois cohomology of commutative group schemes over local and global fields are proven using the technique of satellites. Though some of his results are certainly not unrelated to ours, they seem to give the same statements only in the classical case of abelian varieties.

We thank Yves André, Jean-Louis Colliot-Thélène, Ofer Gabber, Joost van Hamel, Bruno Kahn, Niranjan Ramachandran and Michael Spiess for pertinent comments. The referee's remarks were very helpful for us. Needless to say, we owe a considerable intellectual debt to the work of Tate, Artin/Verdier, Milne and others rendered in [16. A major part of this work was done during the second author's stay at the Université de Paris-Sud whose generous hospitality is gratefully acknowledged. The second author was also partially supported by OTKA grants Nos. T-031984 and F-032325.

\section{Some notation and conventions}

Let $B$ be an abelian group. For each integer $n>0, B[n]$ stands for the $n$-torsion subgroup of $B$ and $B_{\text {tors }}$ for the whole torsion subgroup of $B$. We shall often abbreviate the quotient $B / n B$ by $B / n$. For any prime number $\ell$, we denote by $B\{\ell\}$ the $\ell$-primary torsion subgroup of $B$ and by $\bar{B}\{\ell\}$ the quotient of $B\{\ell\}$ by its maximal divisible subgroup. Also, we denote by $B^{(\ell)}$ the $\ell$-adic completion of $B$, i.e. the projective $\operatorname{limit} \lim B / \ell^{n} B$.

For a topological group $B$, we denote by $B^{\wedge}$ the completion of $B$ with respect to open subgroups of finite index (in the discrete case this is the usual profinite completion of $B$ ). We set $B^{D}$ for the group of continuous homomorphisms $B \rightarrow \mathbf{Q} / \mathbf{Z}$ (in the discrete case these are just all homomorphisms). 
We equip $B^{D}$ with the compact-open topology. The topological group $B$ is compactly generated if $B$ contains a compact subset $K$ such that $K$ generates $B$ as a group. A continuous morphism $f: B \rightarrow C$ of topological groups is strict if the image of any open subset of $B$ is an open subset of $\operatorname{Im} f$ for the topology induced by $C$.

\section{Preliminaries on 1-motives}

Let $S$ be a scheme. Denote by $\mathcal{F}_{S}$ the category of fppf sheaves of abelian groups over $S$ (when $S=\operatorname{Spec} K$ is the spectrum of a field, we shall write $\mathcal{F}_{K}$ for $\left.\mathcal{F}_{S}\right)$. Write $\mathcal{C}^{b}\left(\mathcal{F}_{S}\right)$ for the category of bounded complexes of fppf sheaves over $S$ and $\mathcal{D}^{b}\left(\mathcal{F}_{S}\right)$ for the associated derived category. Recall (e.g. from [22]) that a 1-motive $M$ over $S$ consists of the following data:

- An $S$-group scheme $Y$ which is étale locally isomorphic to $\mathbf{Z}^{r}$ for some $r \geq 0$.

- A commutative $S$-group scheme $G$ fitting into an exact sequence of $S$-groups

$$
0 \rightarrow T \rightarrow G \stackrel{p}{\rightarrow} A \rightarrow 0
$$

where $T$ is an $S$-torus and $A$ an abelian scheme over $S$.

- An $S$-homomorphism $u: Y \rightarrow G$.

The 1-motive $M$ can be viewed as a complex of fppf $S$-sheaves $[Y \stackrel{u}{\rightarrow} G]$, with $Y$ put in degree -1 and $G$ in degree 0 , and also as an object of the derived category $\mathcal{D}^{b}\left(\mathcal{F}_{S}\right)$. It is equipped with a 3 -term weight filtration: $W_{i}(M)=0$ for $i \leq-3, W_{-2}(M)=[0 \rightarrow T], W_{-1}(M)=[0 \rightarrow G]$ and $W_{i}(M)=M$ for $i \geq 0$. From this we shall only need the 1 -motive $M / W_{-2}(M)$, i.e. the complex $[Y \stackrel{h}{\rightarrow} A]$, where $h=p \circ u$. By [22, Proposition 2.3.1, we can identify morphisms of 1-motives in $\mathcal{C}^{b}\left(\mathcal{F}_{S}\right)$ and $\mathcal{D}^{b}\left(\mathcal{F}_{S}\right)$.

To each 1-motive $M$ one can associate a Cartier dual $M^{*}$ by the following construction which we briefly recall. Denote by $Y^{*}$ the group of characters of $T$, by $A^{*}$ the abelian scheme dual to $A$, and by $T^{*}$ the $S$-torus with character group $Y$. According to the generalised Barsotti-Weil formula ([19], III.18), $A^{*}$ represents the functor $S^{\prime} \mapsto \operatorname{Ext}_{S^{\prime}}^{1}\left(A, \mathbf{G}_{m}\right)$ on $\mathcal{F}_{S}$. Writing $M^{\prime}=M / W_{-2} M$, one deduces from this that the functor $S^{\prime} \mapsto \operatorname{Ext}_{S^{\prime}}^{1}\left(M^{\prime}, \mathbf{G}_{m}\right)$ on $\mathcal{C}^{b}\left(\mathcal{F}_{S}\right)$ is representable by an $S$-group scheme $G^{*}$ which is an extension of $A^{*}$ by $T^{*}$. One calls the 1 -motive $\left[0 \rightarrow G^{*}\right]$ the (Cartier) dual of $M^{\prime}$. Pulling back the Poincaré biextension ([16], p. 395) on $A \times A^{*}$ to $A \times G^{*}$ one gets a biextension 
$\mathcal{P}^{\prime}$ of the 1 -motives $M^{\prime}$ and $\left[0 \rightarrow G^{*}\right]$ by $\mathbf{G}_{m}$, which is a $\mathbf{G}_{m}$-torsor over $A \times G^{*}$ whose pullback to $Y \times G^{*}$ by the natural map $Y \times G^{*} \rightarrow A \times G^{*}$ is trivial (cf. 3], 10.2.1 for this definition).

To treat the general case, consider $M$ as an extension of $M^{\prime}$ by $T$. Any element of $Y^{*}=\operatorname{Hom}_{S}\left(T, \mathbf{G}_{m}\right)$ then induces by pushout an extension of $M^{\prime}$ by $\mathbf{G}_{m}$, i.e. an element of $G^{*}$. Whence a map $Y^{*} \rightarrow G^{*}$ which is in fact a map of $S$-group schemes; we call the associated 1-motive $M^{*}$ the (Cartier) dual of $M$. The pullback $\mathcal{P}$ of the biextension $\mathcal{P}^{\prime}$ from $A \times G^{*}$ to $G \times G^{*}$ becomes trivial over $G \times Y^{*}$ when pulled back by the map $G \times Y^{*} \rightarrow G \times G^{*}$, hence defines a biextension of $M$ and $M^{*}$ by $\mathbf{G}_{m}$ (again in the sense of [3]).

According to the formula

$$
\operatorname{Biext}_{S}\left(M, M^{*}, \mathbf{G}_{m}\right) \cong \operatorname{Hom}_{\mathcal{D}^{b}\left(\mathcal{F}_{S}\right)}\left(M \otimes \mathbf{L} M^{*}, \mathbf{G}_{m}[1]\right)
$$

of $(\underline{3}, 10.2 .1)$, the biextension $\mathcal{P}$ defines a map

$$
\Phi_{M}: M \otimes^{\mathbf{L}} M^{*} \rightarrow \mathbf{G}_{m}[1]
$$

in $\mathcal{D}^{b}\left(\mathcal{F}_{S}\right)$, whence pairings

$$
\mathbf{H}^{i}(S, M) \times \mathbf{H}^{j}\left(S, M^{*}\right) \rightarrow H^{i+j+1}\left(S, \mathbf{G}_{m}\right)
$$

for each $i, j \geq 0$.

(Except when explicitly specified, the cohomology groups in this paper are relative to the étale topology; here we can work with either the étale or the fppf topology because $G$ is smooth over $S$ and $Y$ is étale locally constant).

Remark 1.1 Two special cases of this construction are classical :

- $Y=A=0, M=[0 \rightarrow T], M^{*}=Y^{*}[1]$. Then the pairing (2) is just the cup-product $H^{r}(S, T) \times H^{s+1}\left(S, Y^{*}\right) \rightarrow H^{r+s+1}\left(S, \mathbf{G}_{m}\right)$. (Similarly for $T=A=0, M=Y[1], M^{*}=T^{*}$.)

- $Y=T=0, M=[0 \rightarrow A], M^{*}=\left[0 \rightarrow A^{*}\right]$. Then (2) is the wellknown pairing in the cohomology of abelian varieties coming from the generalised Barsotti-Weil formula (compare [16, p. 243 and Chapter III, Appendix C).

Recall finally ([3], 10.1 .5 and 10.1.10) that for any integer $n$ invertible on $S$ and 1-motive $M$ one has an " $n$-adic realisation", namely the finite sheaf (or group scheme) defined by

$$
T_{\mathbf{Z} / n \mathbf{Z}}(M)=H^{0}\left(M[-1] \otimes^{\mathbf{L}} \mathbf{Z} / n \mathbf{Z}\right),
$$


which can be explicitly calculated using the flat resolution $[\mathbf{Z} \stackrel{n}{\rightarrow} \mathbf{Z}]$ of $\mathbf{Z} / n \mathbf{Z}$. The pairing (11) then induces a perfect pairing

$$
T_{\mathbf{Z} / n \mathbf{Z}}(M) \otimes T_{\mathbf{Z} / n \mathbf{Z}}\left(M^{*}\right) \rightarrow \mu_{n}
$$

where $\mu_{n}$ is the sheaf of $n$-th roots of unity. The classical case is when $M$ is of the form $[0 \rightarrow A]$ with $A$ an abelian variety, where we find the well-known Weil pairing.

We finish this section by introducing some notation: for each prime number $\ell$ invertible on $S$, we denote by $T(M)\{\ell\}$ the direct limit of the $T_{\mathbf{Z} / \ell^{n} \mathbf{Z}}(M)$ over all $n>0$ and by $T_{\ell}(M)$ their inverse limit. The piece of notation $T(M)_{\text {tors }}$ stands for the direct sum (taken over all primes $\ell$ invertible on $S$ ) of the groups $T_{\mathbf{Z} / \ell^{n} \mathbf{Z}}(M)$.

\section{Local results}

In this section $S$ is the spectrum of a field $K$, complete with respect to a discrete valuation and with finite residue field. In particular $K$ is a $p$-adic field if char $K=0$, and is isomorphic to the field $\mathbf{F}_{q}((t))$ for some finite field $\mathbf{F}_{q}$ if char $K>0$. We let $\mathcal{O}_{K}$ denote the ring of integers of $K$ and $\mathbf{F}$ its residue field.

Lemma 2.1 For a 1-motive $M=[Y \rightarrow G]$ over $K$, we have

- $\mathbf{H}^{-1}(K, M) \cong \operatorname{Ker}\left[H^{0}(K, Y) \rightarrow H^{0}(K, G)\right]$, a finitely generated free abelian group;

- $\mathbf{H}^{2}(K, M) \cong \operatorname{Coker}\left[H^{2}(K, Y) \rightarrow H^{2}(K, G)\right]$;

- $\mathbf{H}^{i}(K, M)=0 \quad i \neq-1,0,1,2$.

Proof : $\quad$ The field $K$ has strict Galois cohomological dimension 2 ([16], I.1.12). Since $G$ is smooth, $H^{i}(K, G)=0$ for any $i>2$; by [16], I.2.1, we also have $H^{i}(K, Y)=0$ for $i>2$, whence the last equality. For the first two, use moreover the distinguished triangle

$$
Y \rightarrow G \rightarrow M \rightarrow Y[1]
$$

in $\mathcal{C}^{b}\left(\mathcal{F}_{K}\right)$.

Using the trace isomorphism $H^{2}\left(K, \mathbf{G}_{m}\right) \cong \mathbf{Q} / \mathbf{Z}$ of local class field theory, the pairing (2) of the previous section induces bilinear pairings

$$
\mathbf{H}^{i}(K, M) \times \mathbf{H}^{1-i}\left(K, M^{*}\right) \rightarrow \mathbf{Q} / \mathbf{Z}
$$


for all integers $i$ (by the previous lemma, they are trivial for $i \neq-1,0,1,2$ ).

For $i=-1,1,2$, we endow the group $\mathbf{H}^{i}(K, M)$ with the discrete topology. To topologize $\mathbf{H}^{0}(K, M)$ we proceed as follows. The exact triangle (41) yields an exact sequence of abelian groups

$$
0 \rightarrow L \rightarrow G(K) \rightarrow \mathbf{H}^{0}(K, M) \rightarrow H^{1}(K, Y) \rightarrow H^{1}(K, G)
$$

where $L:=H^{0}(K, Y) / \mathbf{H}^{-1}(K, M)$ is a discrete abelian group of finite type. We equip $I=G(K) / \operatorname{Im}(L)$ with the quotient topology (note that in general it is not Hausdorff). The cokernel of the map $G(K) \rightarrow \mathbf{H}^{0}(K, M)$ being finite (as $H^{1}(K, Y)$ itself is finite by [23], II.5.8 iii)), we can define a natural topology on $\mathbf{H}^{0}(K, M)$ by taking as a basis of open neighbourhoods of zero the open neighbourhoods of zero in $I$ (this makes $I$ an open subgroup of finite index of $\left.\mathbf{H}^{0}(K, M)\right)$.

Already in the classical duality theorem for tori over local fields one has to take the profinite completion on $H^{0}$ in order to obtain a perfect pairing. However, for the generalisations we have in mind a nuisance arises from the fact that the completion functor is not always left exact, even if one works only with discrete lattices and $p$-adic Lie groups. As a simple example, consider $K=\mathbf{Q}_{p}(p \geq 3)$ and the injection $\mathbf{Z} \hookrightarrow \mathbf{Q}_{p}^{\times}$given by sending 1 to $1+p$. Here the induced map on completions $\widehat{\mathbf{Z}} \rightarrow\left(\mathbf{Q}_{p}^{\times}\right)^{\wedge}$ is not injective (because $\mathbf{Q}_{p}^{\times} \simeq \mathbf{Z} \times \mathbf{F}_{p}^{\times} \times \mathbf{Z}_{p}$ and the image of $\mathbf{Z}$ lands in the $\mathbf{Z}_{p}$-component).

Bearing this in mind, for a 1-motive $M=[Y \rightarrow G]$ we denote by $\mathbf{H}_{\wedge}^{-1}(K, M)$ the kernel of the map $H^{0}(K, Y)^{\wedge} \rightarrow H^{0}(K, G)^{\wedge}$ coming from $Y \rightarrow G$. There is always an injection $\mathbf{H}^{-1}(K, M)^{\wedge} \rightarrow \mathbf{H}_{\wedge}^{-1}(K, M)$ but it is not an isomorphism in general; the previous example comes from the 1motive $\left[\mathbf{Z} \rightarrow \mathbf{G}_{m}\right]$.

However, we shall also encounter a case where the completion functor behaves well.

Lemma 2.2 Let $G$ be a semi-abelian variety over the local field $K$, with abelian quotient $A$ and toric part $T$. Then the natural sequence

$$
0 \rightarrow T(K)^{\wedge} \rightarrow G(K)^{\wedge} \rightarrow A(K)^{\wedge} \rightarrow H^{1}(K, T)^{\wedge}
$$

is exact. Moreover, $G(K) \hookrightarrow G(K)^{\wedge}$ and $\left(G(K)^{\wedge}\right)^{D}=G(K)^{D}$.

Here in fact we have $A(K)^{\wedge}=A(K)$ (the group $A(K)$ being compact and completely disconnected, hence profinite) and $H^{1}(K, T)^{\wedge}=H^{1}(K, T)$ by finiteness of $H^{1}(K, T)([16]$, I.2.3). 
Proof : To begin with, the maps between completions are well defined because the maps $T(K) \rightarrow G(K), G(K) \rightarrow A(K)$, and $A(K) \rightarrow H^{1}(K, T)$ are continuous (by [13], I.2.1.3, T(K) is closed in $G(K)$ and the image of $G(K)$ is open in $A(K)$ by the implicit function theorem). The theory of Lie groups over a local field shows that $G(K)$ is locally compact, completely disconnected, and compactly generated; we conclude with the third part of the proposition proven in the appendix.

Now we can state the main result of this section.

Theorem 2.3 Let $M=[Y \rightarrow G]$ be a 1-motive over the local field $K$. The pairing (5) induces a perfect pairing between

1. the profinite group $\mathbf{H}_{\wedge}^{-1}(K, M)$ and the discrete group $\mathbf{H}^{2}\left(K, M^{*}\right)$;

2. the profinite group $\mathbf{H}^{0}(K, M)^{\wedge}$ and the discrete group $\mathbf{H}^{1}\left(K, M^{*}\right)$.

In the special cases $M=[0 \rightarrow T]$ or $M=[Y \rightarrow 0]$ we recover Tate-Nakayama duality for tori over $K$ ([23], II.5.8 and [16], I.2.3 for the positive characteristic case) and in the case $M=[0 \rightarrow A]$ we recover Tate's $p$-adic duality theorem for abelian varieties and its generalisation to the positive characteristic case due to Milne ([28], [16], Cor. I.3.4, and Theorem III.7.8).

Proof : For the first statement, set $M^{\prime}:=M / W_{-2} M$. The dual of $M^{\prime}$ is of the form $\left[0 \rightarrow G^{*}\right]$, where $G^{*}$ is an extension of $A^{*}$ by $T^{*}$. Via the pairing (5) for $i=-1,0$, we obtain a commutative diagram

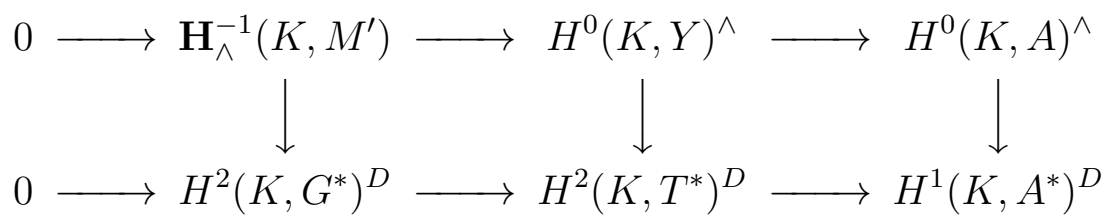

The first line of this diagram is exact by definition, and the second one is exact because it is the dual of an exact sequence of discrete groups (recall that $H^{2}\left(K, A^{*}\right)=0$ by [23, II.5.3, Prop. 16 and [16], III.7.8). By Tate duality for abelian varieties and Tate-Nakayama duality for tori, the last two vertical maps are isomorphisms, hence the same holds for the first one.

Now using Lemma 2.2 we get that the map $H^{0}(K, Y) \rightarrow H^{0}(K, G)$ induces a map $\mathbf{H}_{\wedge}^{-1}\left(K, M^{\prime}\right) \rightarrow T(K)^{\wedge}$ with kernel $\mathbf{H}_{\wedge}^{-1}(K, M)$. ¿From the definition of $M^{\prime}$ we get a commutative diagram with exact rows

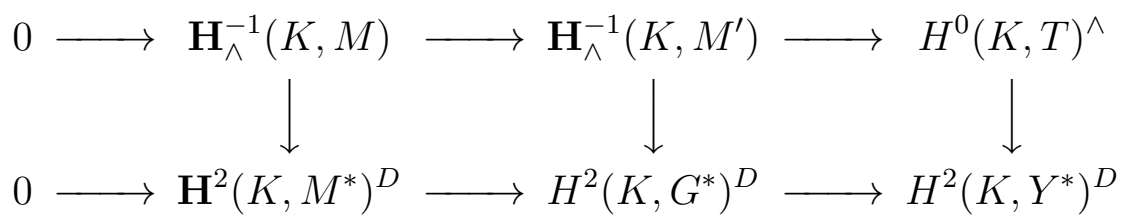


whence we conclude as above that the left vertical map is an isomorphism, by the first part and Tate-Nakayama duality. Then $H^{2}\left(K, M^{*}\right) \cong H_{\wedge}^{-1}(K, M)^{D}$ follows by dualising, using the isomorphism $H^{2}\left(K, M^{*}\right)^{D D} \cong H^{2}\left(K, M^{*}\right)$ for the discrete torsion group $H^{2}\left(K, M^{*}\right)$.

For the second statement, we also begin by working with $M^{\prime}$. Using the pairings (51) and Lemma 2.2 (applied to $G^{*}$ ), we get a commutative diagram with exact rows:

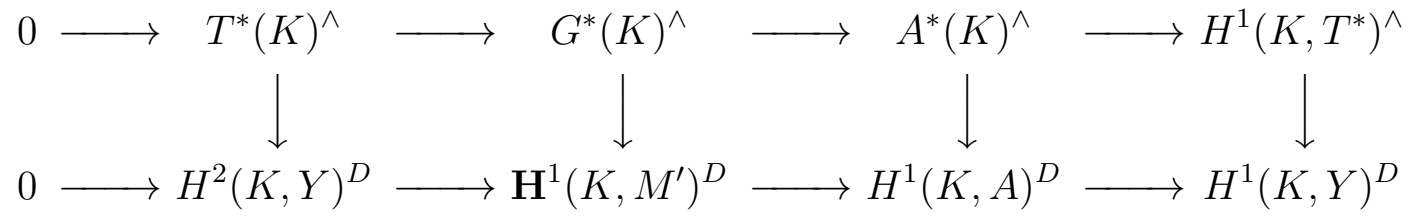

Using the local dualities for $\left(A, A^{*}\right)$ and $\left(Y, T^{*}\right)$, this implies that the map $G^{*}(K)^{\wedge} \rightarrow \mathbf{H}^{1}\left(K, M^{\prime}\right)^{D}$ is an isomorphism.

Now the distinguished triangle $T \rightarrow M \rightarrow M^{\prime} \rightarrow T[1]$ in $\mathcal{C}^{b}\left(\mathcal{F}_{K}\right)$ induces the following commutative diagram with exact rows:

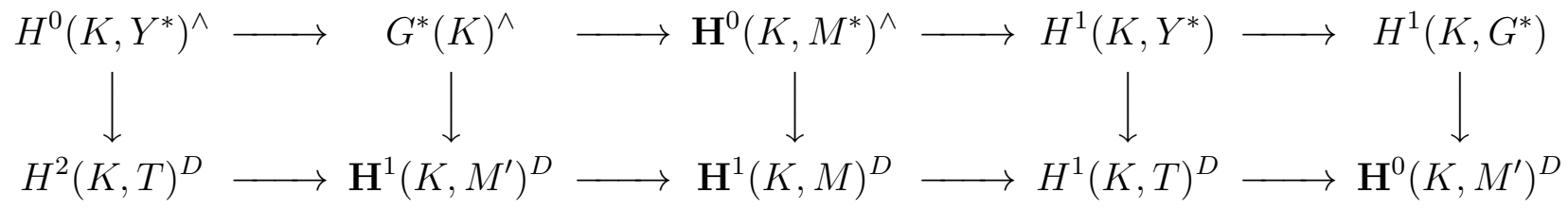

Here the exactness of the rows needs some justification. The upper row is exact without completing the first three terms. Completion in the third term is possible by finiteness of the fourth, and completion in the first two terms is possible because the map $G^{*}(K) \rightarrow \mathbf{H}^{0}\left(K, M^{*}\right)$ is open with finite cokernel by definition of the topology on the target. In the lower row dualisation behaves well because the first four terms are duals of discrete torsion groups.

By Tate-Nakayama duality for tori and what we have already proven, the first, second and fourth vertical maps are isomorphisms. To derive an isomorphism in the middle it remains to prove the injectivity of the fifth map.

This in turn follows from the commutative diagram with exact rows (where again we have used the finiteness of $H^{1}\left(K, T^{*}\right)$ and of $H^{1}(K, Y)$ ):

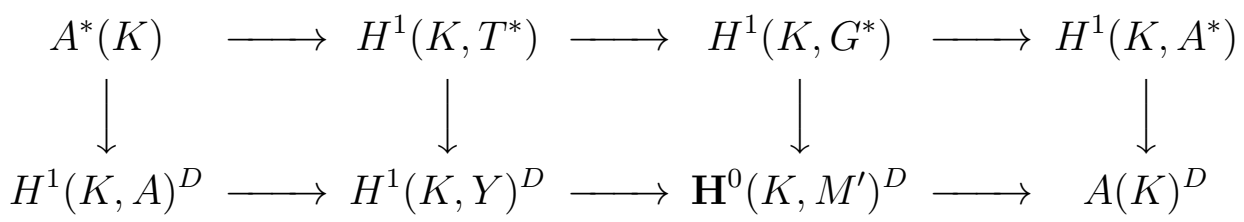


Here the first, second and fourth vertical maps are isomorphisms by local duality for tori and abelian varieties. Again, exactness at the third term of the lower row follows from the definition of the topology on $\mathbf{H}^{0}\left(K, M^{\prime}\right)$. Finally the map $\mathbf{H}^{0}\left(K, M^{*}\right)^{\wedge} \rightarrow \mathbf{H}^{1}(K, M)^{D}$ is an isomorphism and applying this statement to $M^{*}$ instead of $M$, we obtain the theorem.

Remark 2.4 If $K$ is of characteristic zero, any subgroup of finite index of $T(K)$ is open (cf. [16, p.32). It is easy to see that in this case $\mathbf{H}^{0}(K, M)^{\wedge}$ is just the profinite completion of $\mathbf{H}^{0}(K, M)$.

Next we state a version of Theorem 2.3 for henselian fields that will be needed for the global theory.

Theorem 2.5 Let $F$ be the field of fractions of a henselian discrete valuation ring $R$ with finite residue field and let $M$ be a 1-motive over $F$. Assume that $F$ is of characteristic zero. Then the pairing (5) induces perfect pairings

$$
\begin{aligned}
& \mathbf{H}_{\wedge}^{-1}(F, M) \times \mathbf{H}^{2}\left(F, M^{*}\right) \rightarrow \mathbf{Q} / \mathbf{Z} \\
& \mathbf{H}^{0}(F, M)^{\wedge} \times \mathbf{H}^{1}\left(F, M^{*}\right) \rightarrow \mathbf{Q} / \mathbf{Z}
\end{aligned}
$$

where $\mathbf{H}_{\wedge}^{-1}(F, M):=\operatorname{Ker}\left[H^{0}(F, Y)^{\wedge} \rightarrow G(F)^{\wedge}\right]$ and ${ }^{\wedge}$ means profinite completion.

\section{Remarks 2.6}

1. Denoting by $K$ the completion of $F$, the group $\mathbf{H}^{0}(F, M)$ injects into $\mathbf{H}^{0}(K, M)$ by the lemma below, hence it is natural to equip $\mathbf{H}^{0}(F, M)$ with the topology induced by $\mathbf{H}^{0}(K, M)$. But we shall also show that $\mathbf{H}^{0}(F, M)$ and $\mathbf{H}^{0}(K, M)$ have the same profinite completion, hence by Remark 2.4 the profinite completion of $\mathbf{H}^{0}(F, M)$ coincides with its completion with respect to open subgroups of finite index. Therefore there is no incoherence in the notation.

2. In characteristic $p>0$, the analogue of Theorem 2.5 is not clear because of the $p$-part of the groups. Compare [16], III.6.13.

Taking the first remark into account, Theorem 2.5 immediately results from Theorem 2.3 via the following lemma.

Lemma 2.7 Keeping the assumptions of the theorem, denote by $K$ the completion of $F$. Then the natural map $\mathbf{H}^{i}(F, M) \rightarrow \mathbf{H}^{i}(K, M)$ is an injection for $i=0$ inducing an isomorphism $\mathbf{H}^{0}(F, M)^{\wedge} \rightarrow \mathbf{H}^{0}(K, M)^{\wedge}$ on completions, and an isomorphism for $i \geq 1$. 
Proof : For any $n>0$, the canonical map $G(F) / n \rightarrow G(K) / n$ is surjective, for $G(F)$ is dense in $G(K)$ by Greenberg's approximation theorem 7, and $n G(K) \subset G(K)$ is an open subgroup. But this map is also injective, for any point $P \in G(K)$ with $n P \in G(F)$ is locally given by coordinates algebraic over $F$, but $F$ is algebraically closed in $K$ (apply e.g. [17, Theorem 4.11.11 and note that $F$ is of characteristic 0 ), hence $P \in G(F)$. Since $Y$ is locally constant in the étale topology over $\operatorname{Spec} F$, we have $H^{i}(F, Y)=H^{i}(K, Y)$ for each $i \geq 0$. The case $i=0$ of the lemma follows from these facts by dévissage.

To treat the cases $i>0$, recall first that multiplication by $n$ on $G$ is surjective in the étale topology. Therefore

$$
H^{i}(F, G)[n]=\operatorname{coker}\left[H^{i-1}(F, G) / n \rightarrow H^{i}(F, G[n])\right]
$$

for $i \geq 1$, and similarly for $H^{i}(K, G)$. Moreover, $H^{i}(F, G[n])=H^{i}(K, G[n])$ because $G[n]$ is locally constant in the étale topology (note that $F$ and $K$ have the same absolute Galois group). Starting from the isomorphism $G(F) / n \cong G(K) / n$ already proven, we thus obtain isomorphisms of torsion abelian groups $H^{i}(F, G) \simeq H^{i}(K, G)$ for any $i \geq 1$ by induction on $i$, which together with the similar isomorphisms for $Y$ mentioned above yield the statement by dévissage.

We shall also need the following slightly finer statement.

Proposition 2.8 Keeping the notations above, equip $\mathbf{H}^{0}\left(F, M^{*}\right)$ with the topology induced by $\mathbf{H}^{0}\left(K, M^{*}\right)$. Then $\mathbf{H}^{0}\left(F, M^{*}\right)$ injects into $\mathbf{H}^{0}\left(F, M^{*}\right)^{\wedge}$ and they have the same continuous dual. Moreover, the pairing (5) yields an isomorphism

$$
\mathbf{H}^{1}(F, M) \cong \mathbf{H}^{0}\left(F, M^{*}\right)^{D} .
$$

Proof : For the first statement we use the exact sequence

$$
H^{0}\left(F, Y^{*}\right) \rightarrow G^{*}(F) \rightarrow \mathbf{H}^{0}\left(F, M^{*}\right) \rightarrow H^{1}\left(F, Y^{*}\right) \rightarrow H^{1}\left(F, G^{*}\right)
$$

and similarly for $K$ instead of $F$. By definition of the topology on $\mathbf{H}^{0}\left(F, M^{*}\right)$ and $\mathbf{H}^{0}\left(K, M^{*}\right)$, the duals of these sequences remain exact thanks to the result proven in the Appendix. The group $G^{*}(F)$ injects into $G(K)^{\wedge}$ by Lemma 2.7 and Lemma 2.2 moreover, since $G^{*}(F)$ is a dense subgroup of $G^{*}(K)$, they have the same dual. Similarly, we have an injection $H^{0}\left(F, Y^{*}\right) \hookrightarrow$ $H^{0}\left(K, Y^{*}\right)^{\wedge}$ using that $H^{0}\left(K, Y^{*}\right)$ is of finite type, and this map induces an isomorphism on duals. Thus the first statement follows from the exact sequence using the finiteness of $H^{1}\left(F, Y^{*}\right) \hookrightarrow H^{1}\left(K, Y^{*}\right)$ and Lemma 2.7. The second statement now follows from Theorem [2.3, again using Lemma 2.7 
For the global case, we shall also need a statement for the real case. Consider a 1-motive $M_{\mathbf{R}}$ over the spectrum of the field $\mathbf{R}$ of real numbers. As in the classical cases, the duality results in the previous section extend in a straightforward fashion to this situation, provided that we replace usual Galois cohomology groups by Tate modified groups. Denote by $\Gamma_{\mathbf{R}}=\operatorname{Gal}(\mathbf{C} / \mathbf{R}) \simeq \mathbf{Z} / 2$ the Galois group of $\mathbf{R}$. Let $\mathcal{F}^{\bullet}$ be a bounded complex of $\mathbf{R}$-groups. For each $i \in \mathbf{Z}$, the modified hypercohomology groups $\widehat{\mathbf{H}}^{i}\left(\mathbf{R}, \mathcal{F}^{\bullet}\right)$ are defined in the usual way: for each term $\mathcal{F}^{i}$ of $\mathcal{F}$, we take the standard Tate complex associated to the $\Gamma_{\mathbf{R}}$-module $\mathcal{F}^{i}(\mathbf{C})$ (cf. [16], pp. 2$3)$; then we obtain Tate hypercohomology groups via the complex associated to the arising double complex. From the corresponding well-known results in Galois cohomology, it is easy to see that $\widehat{\mathbf{H}}^{i}\left(\mathbf{R}, \mathcal{F}^{\bullet}\right)=\mathbf{H}^{i}\left(\mathbf{R}, \mathcal{F}^{\bullet}\right)$ for $i \geq 1$ if $\mathcal{F}^{\bullet}$ is concentrated in nonpositive degrees, and that $\widehat{H}^{i}\left(\mathbf{R}, \mathcal{F}^{\bullet}\right)$ is isomorphic to $\widehat{\mathbf{H}}^{i+2}\left(\mathbf{R}, \mathcal{F}^{\bullet}\right)$ for any $i \in \mathbf{Z}$. Recall also that the Brauer group $\operatorname{Br} \mathbf{R}$ is isomorphic to $\mathbf{Z} / 2 \mathbf{Z} \subset \mathbf{Q} / \mathbf{Z}$ via the local invariant.

Now we have the following analogue of Theorem 2.3 .

Proposition 2.9 Let $M_{\mathbf{R}}=\left[Y_{\mathbf{R}} \rightarrow G_{\mathbf{R}}\right]$ be a 1-motive over $\mathbf{R}$. Then the cup-product pairing induces a perfect pairing of finite 2-torsion groups

$$
\widehat{\mathbf{H}}^{0}\left(\mathbf{R}, M_{\mathbf{R}}\right) \times \widehat{\mathbf{H}}^{1}\left(\mathbf{R}, M_{\mathbf{R}}^{*}\right) \rightarrow \mathbf{Z} / 2 \mathbf{Z}
$$

Proof : Let $T_{\mathbf{R}}$ (resp. $A_{\mathbf{R}}$ ) be the torus (resp. the abelian variety) corresponding to $G_{\mathbf{R}}$. In the special cases $M_{\mathbf{R}}=T_{\mathbf{R}}, M_{\mathbf{R}}=A_{\mathbf{R}}, M_{\mathbf{R}}=Y_{\mathbf{R}}[1]$, the result is known ([16], I.2.13 and I.3.7). Now the proof by devissage consists exactly of the same steps as in Theorem 2.3, except that we don't have to take any profinite completions, all occurring groups being finite.

In Section 5 we shall need the fact that when $M$ is a 1-motive over a local field $K$ which extends to a 1-motive over $\operatorname{Spec} \mathcal{O}_{K}$, the unramified parts of the cohomology are exact annihilators of each other in the local duality pairing for $i=1$ (see [23], II.5.5, [18], Theorem 7.2.15 and [16], III.1.4 for analogues for finite modules). More precisely, let $\mathcal{M}=[\mathcal{Y} \rightarrow \mathcal{G}]$ be a 1 motive over $\operatorname{Spec} \mathcal{O}_{K}$ and $M=[Y \rightarrow G]$ the restriction of $\mathcal{M}$ to Spec $K$. Denote by $\mathbf{H}_{\mathrm{nr}}^{0}(K, M)$ and $\mathbf{H}_{\mathrm{nr}}^{1}\left(K, M^{*}\right)$ the respective images of the maps $\mathbf{H}^{0}\left(\mathcal{O}_{K}, \mathcal{M}\right) \rightarrow \mathbf{H}^{0}(K, M)$ and $\mathbf{H}^{1}\left(\mathcal{O}_{K}, \mathcal{M}^{*}\right) \rightarrow \mathbf{H}^{1}\left(K, M^{*}\right)$. To make the notation simpler, we still let $\mathbf{H}_{\mathrm{nr}}^{0}(K, M)^{\wedge}$ denote the image of $\mathbf{H}_{\mathrm{nr}}^{0}(K, M)^{\wedge}$ in $\mathbf{H}^{0}(K, M)^{\wedge}$. (We work with complete fields since this is what will be needed later; the henselian case is similar in mixed characteristic.) 
Theorem 2.10 In the above situation, $\mathbf{H}_{\mathrm{nr}}^{0}(K, M)^{\wedge}$ and $\mathbf{H}_{\mathrm{nr}}^{1}\left(K, M^{*}\right)$ are the exact annihilators of each other in the pairing

$$
\mathbf{H}^{0}(K, M)^{\wedge} \times \mathbf{H}^{1}\left(K, M^{*}\right) \rightarrow \mathbf{Q} / \mathbf{Z}
$$

induced by (5).

Proof : The restriction of the local pairing to $\mathbf{H}_{\mathrm{nr}}^{0}(K, M) \times \mathbf{H}_{\mathrm{nr}}^{1}\left(K, M^{*}\right)$ is zero because $H^{2}\left(\mathcal{O}_{K}, \mathbf{G}_{m}\right) \cong H^{2}\left(\mathbf{F}, \mathbf{G}_{m}\right)=0$. Thus it is sufficient to show that the maps

$$
\begin{aligned}
\mathbf{H}^{0}(K, M)^{\wedge} / \mathbf{H}_{\mathrm{nr}}^{0}(K, M)^{\wedge} & \rightarrow \mathbf{H}_{\mathrm{nr}}^{1}\left(K, M^{*}\right)^{D}, \\
\mathbf{H}^{1}\left(K, M^{*}\right) / \mathbf{H}_{\mathrm{nr}}^{1}\left(K, M^{*}\right) & \rightarrow \mathbf{H}_{\mathrm{nr}}^{0}(K, M)^{D}
\end{aligned}
$$

are injective, where we have equipped $\mathbf{H}_{\mathrm{nr}}^{1}\left(K, M^{*}\right)$ with the discrete topology and $\mathbf{H}_{\mathrm{nr}}^{0}(K, M)$ with the topology induced by that on $\mathbf{H}^{0}(K, M)$.

Denote by $\mathcal{T}$ (resp. $T$ ) the torus and by $\mathcal{A}$ (resp. $A$ ) the abelian scheme (resp. abelian variety) corresponding to $\mathcal{G}$ (resp. $G$ ). We need the following lemma presumably well known to the experts.

Lemma 2.11 In the Tate-Nakayama pairing

$$
H^{2}(K, Y) \times H^{0}\left(K, T^{*}\right) \rightarrow \mathbf{Q} / \mathbf{Z}
$$

the exact annihilator of $H_{\mathrm{nr}}^{0}\left(K, T^{*}\right)$ is $H_{\mathrm{nr}}^{2}(K, Y)$.

Proof : Let $n>0$. We work in flat cohomology. The exact sequence of fppf sheaves

$$
0 \rightarrow \mathcal{T}[n] \rightarrow \mathcal{T} \rightarrow \mathcal{T} \rightarrow 0
$$

and the cup-product pairings induce a commutative diagram with exact rows:

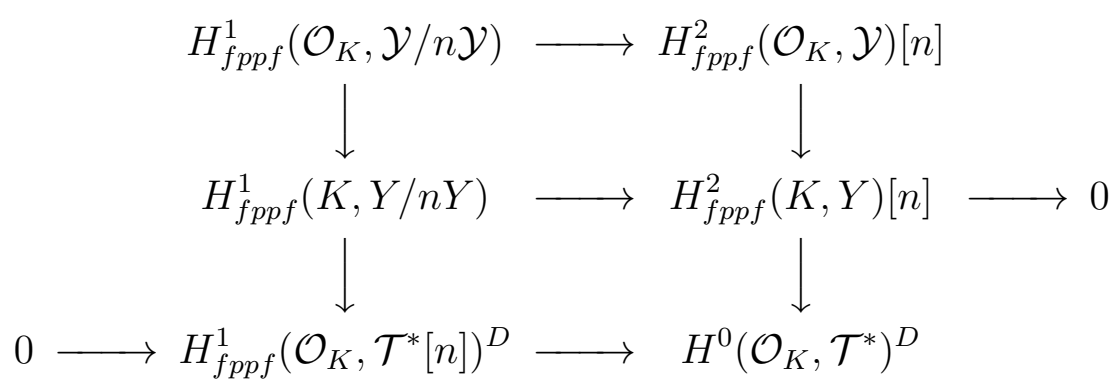

where all groups are given the discrete topology. The zero at lower left comes from the vanishing $H_{f p p f}^{1}\left(\mathcal{O}_{K}, \mathcal{T}^{*}\right) \cong H_{f p p f}^{1}\left(\mathbf{F}, \widetilde{T}^{*}\right)=0$ (where $\widetilde{T}^{*}$ stands for 
the special fibre of $\mathcal{T}^{*}$, which is a consequence of Lang's theorem ([12], Theorem 2 and [15], III.3.11).

Now by [16, III.1.4. and III.7.2., the left column is exact. Therefore the right column is exact as well. To see that this implies the statement it remains to note that since $\mathcal{Y}$ is a smooth group scheme over $\operatorname{Spec} \mathcal{O}_{K}$, its étale and flat cohomology groups are the same and moreover they are all torsion in positive degrees.

We resume the proof of Theorem 2.10. The weight filtration on $M$, the cup-product pairings and the inclusion $\mathcal{O}_{K} \subset K$ induce a commutative diagram with exact rows (here the groups in the lower row are given the discrete topology)

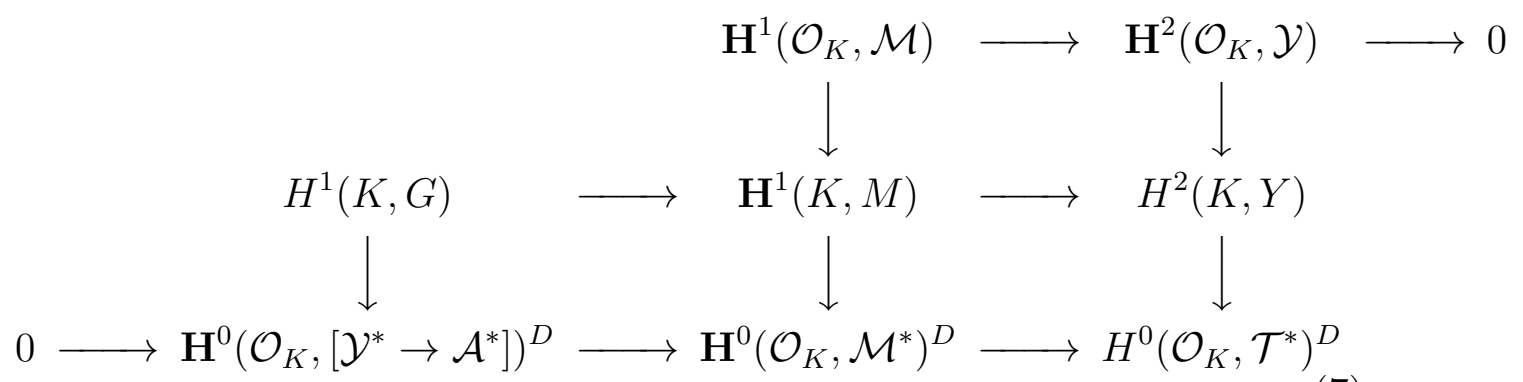

where the two zeros come from the vanishing of the groups $H^{1}\left(\mathcal{O}_{K}, \mathcal{T}^{*}\right)$ and $H^{2}\left(\mathcal{O}_{K}, \mathcal{G}\right)=H^{2}(\mathbf{F}, \widetilde{G})$; the second vanishing follows from the fact that $\widetilde{G}(\overline{\mathbf{F}})$ is torsion and $\mathbf{F}$ is of cohomological dimension 1.

Next, observe that the map $\mathbf{H}^{0}\left(\mathcal{O}_{K},\left[\mathcal{Y}^{*} \rightarrow \mathcal{A}^{*}\right]\right) \rightarrow \mathbf{H}^{0}\left(K,\left[Y^{*} \rightarrow A^{*}\right]\right)$ is an isomorphism. Indeed, by dévissage this reduces to showing that the natural maps $H^{0}\left(\mathcal{O}_{K}, \mathcal{A}\right) \rightarrow H^{0}(K, A)$ and $H^{1}\left(\mathcal{O}_{K}, \mathcal{Y}\right) \rightarrow H^{1}(K, Y)$ are isomorphisms. The first isomorphism follows from the properness of the abelian scheme $\mathcal{A}$. For the second, denote by $\mathcal{O}_{K}^{n r}$ the strict henselisation of $\mathcal{O}_{K}$ and by $K^{n r}$ its fraction field. Then the Hochschild-Serre spectral sequence induces a commutative diagram with exact rows:

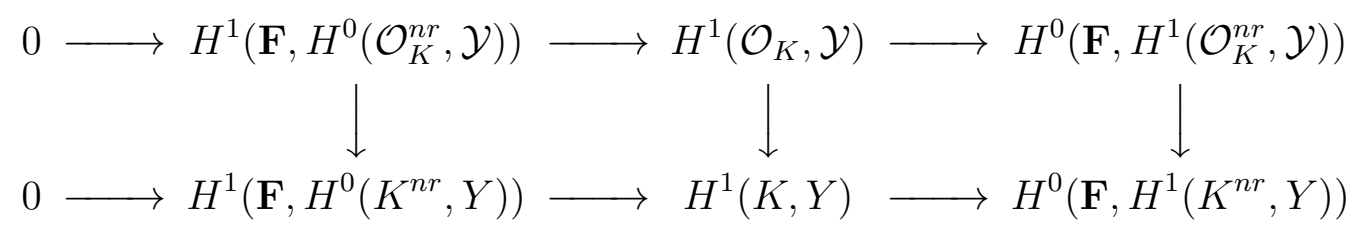

Here the group at top right vanishes because $\mathcal{O}_{K}^{n r}$ is acyclic for étale cohomology. Also, since $\mathcal{O}_{K}^{n r}$ is simply connected for the étale topology, the sheaf $\mathcal{Y}$ is isomorphic to a (torsion free) constant sheaf $\mathbf{Z}^{r}$, whence the vanishing of the group at bottom right. For the same reason, both groups on the left 
are isomorphic to $H^{1}\left(\mathrm{Gal}\left(\mathbf{F}^{\prime} / \mathbf{F}\right), H^{0}\left(\mathcal{O}_{K}^{n r}, \mathcal{Y}\right)\right)$, where $\mathbf{F}^{\prime}$ is a finite extension trivialising the action of $\operatorname{Gal}(\bar{F} \mid F)$ on $H^{0}\left(\mathcal{O}_{K}^{n r}, \mathcal{Y}\right)$, whence the claim.

This being said, we conclude from Proposition 2.8 (which also holds in positive characteristic over complete fields) that the left vertical map in diagram (77) is injective. On the other hand the right column is exact by Lemma 2.11]. Hence the middle column, i.e. the sequence

$$
\mathbf{H}^{1}\left(\mathcal{O}_{K}, M\right) \rightarrow \mathbf{H}^{1}(K, M) \rightarrow \mathbf{H}^{0}\left(\mathcal{O}_{K}, \mathcal{M}^{*}\right)^{D}
$$

is exact. Since the map $\mathbf{H}^{1}(K, M) \rightarrow \mathbf{H}^{0}\left(\mathcal{O}_{K}, \mathcal{M}^{*}\right)^{D}$ factors through the $\operatorname{map} \mathbf{H}^{1}(K, M) \rightarrow\left(\mathbf{H}_{\mathrm{nr}}^{0}\left(K, M^{*}\right)^{\wedge}\right)^{D}$, the sequence

$$
\mathbf{H}^{1}\left(\mathcal{O}_{K}, M\right) \rightarrow \mathbf{H}^{1}(K, M) \rightarrow\left(\mathbf{H}_{\mathrm{nr}}^{0}\left(K, M^{*}\right)^{\wedge}\right)^{D}
$$

(of which we knew before that it is a complex) is exact as well. Dualising this exact sequence of discrete groups, we obtain from Theorem 2.3 that the sequence

$$
\mathbf{H}_{\mathrm{nr}}^{0}\left(K, M^{*}\right)^{\wedge} \rightarrow \mathbf{H}^{0}(K, M)^{\wedge} \rightarrow \mathbf{H}^{1}\left(\mathcal{O}_{K}, M\right)^{D}
$$

is exact and the theorem is proven.

\section{Global results : étale cohomology}

Let $k$ be a number field with ring of integers $\mathcal{O}_{k}$. Denote by $\Omega_{k}$ the set of places of $k$ and $\Omega_{k}^{\infty} \subset \Omega_{k}$ the subset of real places. Let $k_{v}$ be the completion of $k$ at $v$ if $v$ is archimedean, and the field of fractions of the henselisation of the local ring of $\operatorname{Spec} \mathcal{O}_{k}$ at $v$ if $v$ is finite. In the latter case, the piece of notation $\hat{k}_{v}$ stands for the completion of $k$ at $v$. We denote by $U$ an open subscheme of $\operatorname{Spec} \mathcal{O}_{k}$ and by $\Sigma_{f}$ the set of finite places coming from closed points outside $U$.

In this section and the next, every abelian group is equipped with the discrete topology; in particular $B^{\wedge}$ denotes the profinite completion of $B$ and $B^{D}:=\operatorname{Hom}(B, \mathbf{Q} / \mathbf{Z})$ (even if $B$ has a natural nondiscrete topology).

We shall need the notion of "cohomology groups with compact support" $\mathbf{H}_{c}^{i}\left(U, \mathcal{F}^{\bullet}\right)$ for cohomologically bounded complexes of abelian sheaves $\mathcal{F}^{\bullet}$ on $U$. For $k$ totally imaginary, these satisfy $\mathbf{H}_{c}^{i}\left(U, \mathcal{F}^{\bullet}\right)=\mathbf{H}^{i}\left(\operatorname{Spec} \mathcal{O}_{k}, j_{!} \mathcal{F}^{\bullet}\right)$, where $j: U \rightarrow \operatorname{Spec} \mathcal{O}_{k}$ is the inclusion map. In the general case this equality holds up to a finite 2-group. More precisely, there exists a long exact sequence (infinite in both directions)

$$
\cdots \rightarrow \mathbf{H}_{c}^{i}\left(U, \mathcal{F}^{\bullet}\right) \rightarrow \mathbf{H}^{i}\left(U, \mathcal{F}^{\bullet}\right) \rightarrow \bigoplus_{v \in \Sigma_{f}} \mathbf{H}^{i}\left(\hat{k}_{v}, \mathcal{F}^{\bullet}\right) \oplus \bigoplus_{v \in \Omega_{k}^{\infty}} \widehat{\mathbf{H}}^{i}\left(k_{v}, \mathcal{F}^{\bullet}\right) \rightarrow \mathbf{H}_{c}^{i+1}\left(U, \mathcal{F}^{\bullet}\right) \rightarrow \ldots
$$


where for $v \in \Omega_{k}^{\infty}$ the notation $\widehat{\mathbf{H}}^{i}\left(k_{v}, \mathcal{F}^{\bullet}\right)$ stands for Tate (modified) cohomology groups of the group $\operatorname{Gal}\left(\bar{k}_{v} / k_{v}\right) \cong \mathbf{Z} / 2 \mathbf{Z}$ and where we have abused notation in denoting the pullbacks of $\mathcal{F}^{\bullet}$ under the maps $\operatorname{Spec} k_{v} \rightarrow \operatorname{Spec} \mathcal{O}_{k}$ by the same symbol.

In the literature, two constructions for the groups $\mathbf{H}_{c}^{i}\left(U, \mathcal{F}^{\bullet}\right)$ have been proposed, by Kato [9] and by Milne [16], respectively. The two definitions are equivalent (though we could not find an appropriate reference for this fact). We shall use Kato's construction which we find more natural and which we now copy from [9] for the convenience of the reader.

First, for an abelian sheaf $\mathcal{G}$ on the big étale site of $\operatorname{Spec} \mathbf{Z}$, one defines a complex $\widehat{\mathcal{G}}^{\bullet}$ as follows. Denote by $a: \operatorname{Spec} \mathbf{C} \rightarrow$ Spec $\mathbf{Z}$ the canonical morphism and by $\sigma: a_{*} a^{*} \mathcal{G} \rightarrow a_{*} a^{*} \mathcal{G}$ the canonical action of the complex conjugation viewed as an element of $\operatorname{Gal}(\mathbf{C} / \mathbf{R})$. Now put $\widehat{\mathcal{G}}^{0}=\mathcal{G} \oplus a_{*} a^{*} \mathcal{G}$ and $\widehat{\mathcal{G}}^{i}=a_{*} a^{*} \mathcal{G}$ for $i \in \mathbf{Z} \backslash\{0\}$. One defines the differentials $d^{i}$ of the complex $\widehat{\mathcal{G}}^{\bullet}$ as follows:

$$
d^{-1}(x)=(0,(\sigma-\mathrm{id})(x)) ; \quad d^{0}(x, y)=b(x)+(\sigma+\mathrm{id})(y),
$$

where $b: \mathcal{G} \rightarrow a_{*} a^{*} \mathcal{G}$ is the adjunction map; otherwise, set $d^{i}=\sigma+$ id for $i$ even and $d^{i}=\sigma-\mathrm{id}$ for $i$ odd. This definition extends to bounded complexes $\mathcal{G}^{\bullet}$ on the big étale site of $\operatorname{Spec} \mathbf{Z}$ in the usual way: construct the complex $\widehat{\mathcal{G}}^{i}$ for each term $\mathcal{G}^{i}$ of the complex and then take the complex associated to the arising double complex. Finally for $U$ and $\mathcal{F}^{\bullet}$ as above, one sets

$$
\mathbf{H}_{c}^{i}\left(U, \mathcal{F}^{\bullet}\right):=\mathbf{H}^{i}\left(\operatorname{Spec} \mathbf{Z}, \widehat{\mathbf{R} f_{!} \mathcal{F}^{\bullet}}\right),
$$

where $f: U \rightarrow \operatorname{Spec} \mathbf{Z}$ is the canonical morphism.

¿From this definition one infers that for an open immersion $j_{V}: V \rightarrow U$ and a complex $\mathcal{F}_{V}^{\bullet}$ of sheaves on $V$ one has $\mathbf{H}_{c}^{i}\left(V, \mathcal{F}_{V}^{\bullet}\right) \cong \mathbf{H}_{c}^{i}\left(U, j_{V !} \mathcal{F}_{V}^{\bullet}\right)$; therefore, setting $\mathcal{F}_{V}^{\bullet}=j_{V}^{*} \mathcal{F}^{\bullet}$ one obtains a canonical map

$$
\mathbf{H}_{c}^{i}\left(V, \mathcal{F}_{V}^{\bullet}\right) \rightarrow \mathbf{H}_{c}^{i}\left(U, \mathcal{F}^{\bullet}\right)
$$

coming from the morphism of complexes $j_{V !} j_{V}^{*} \mathcal{F}^{\bullet} \rightarrow \mathcal{F}^{\bullet}$. This covariant functoriality for open immersions will be crucial for the arguments in the next section.

Finally, we remark that for cohomologically bounded complexes $\mathcal{F}^{\bullet}$ and $\mathcal{G}^{\bullet}$ of étale sheaves on $U$, one has a cup-product pairing

$$
\mathbf{H}^{i}\left(U, \mathcal{F}^{\bullet}\right) \times \mathbf{H}_{c}^{j}\left(U, \mathcal{G}^{\bullet}\right) \rightarrow \mathbf{H}_{c}^{i+j+1}\left(U, \mathcal{F}^{\bullet} \otimes^{\mathbf{L}} \mathcal{G}^{\bullet}\right)
$$

Indeed, for $f$ as above (which is quasi-finite by definition), one knows from general theorems of étale cohomology that the complexes $\mathbf{R} f_{*} \mathcal{F}^{\bullet}$ and 
$\mathbf{R} f_{!} \mathcal{F}^{\bullet}$ are cohomologically bounded (and similarly for $\mathcal{G}^{\bullet}, \mathcal{F}^{\bullet} \otimes^{\mathbf{L}} \mathcal{G}^{\bullet}$ ), and that there exists a canonical pairing $\mathbf{R} f_{*} \mathcal{F}^{\bullet} \otimes^{\mathbf{L}} \mathbf{R} f_{!} \mathcal{G}^{\bullet} \rightarrow \mathbf{R} f_{!}\left(\mathcal{F}^{\bullet} \otimes^{\mathbf{L}} \mathcal{G}^{\bullet}\right)$. One then uses the simple remark that any derived pairing $\mathcal{A}^{\bullet} \otimes^{\mathbf{L}} \mathcal{B}^{\bullet} \rightarrow \mathcal{C}^{\bullet}$ of cohomologically bounded complexes of étale sheaves on $\operatorname{Spec} \mathbf{Z}$ induces a pairing $\mathcal{A}^{\bullet} \otimes^{\mathbf{L}} \widehat{\mathcal{B}^{\bullet}} \rightarrow \widehat{\mathcal{C}^{\bullet}}$.

Remark 3.1 In [30, Zink defines modified cohomology groups $\widehat{H}^{i}(U, \mathcal{F})$ which take the real places into account and satisfy a localisation sequence for cohomology. He applies this to prove the Artin-Verdier duality theorem for finite sheaves in the case $U=\operatorname{Spec} \mathcal{O}_{k}$ (where cohomology and compact support cohomology coincide). For general $U$, however, one needs the groups $H_{c}^{i}(U, \mathcal{F})$ which are the same as the groups $\widehat{H}^{i}\left(\operatorname{Spec} \mathcal{O}_{k}, j ! \mathcal{F}\right)$ in his notation.

This being said, we return to 1-motives.

Lemma 3.2 Let $M$ be a 1-motive over $U$.

1. The groups $\mathbf{H}^{i}(U, M)$ are torsion for $i \geq 1$ and so are the groups $\mathbf{H}_{c}^{i}(U, M)$ for $i \geq 2$.

2. For any $\ell$ invertible on $U$, the groups $\mathbf{H}^{i}(U, M)\{\ell\}(i \geq 1)$ are of finite cotype. Same assertion for the groups $\mathbf{H}_{c}^{i}(U, M)\{\ell\}(i \geq 2)$.

3. The group $\mathbf{H}^{0}(U, M)$ is of finite type.

Proof : For the first part of (1) note that, with the notation of Section 1. the group $H^{i}(U, A)$ is torsion ([16, II.5.1), and so are $H^{i}(U, T)$ and $H^{i+1}(U, Y)$ by [16], II.2.9. The second part then follows from exact sequence (8) and the local facts.

By this last argument, for (2) it is again enough to prove the first statement. To do so, observe that $H^{i}(U, A)\{l\}$ is of finite cotype ([16, II.5.2) and for each positive integer $n$, there are surjective maps

$$
H^{i}\left(U, Y / l^{n} Y\right) \rightarrow H^{i+1}(U, Y)\left[l^{n}\right], \quad H^{i}\left(U, T\left[l^{n}\right]\right) \rightarrow H^{i}(U, T)\left[l^{n}\right]
$$

whose sources are finite by [16], II.3.1.

To prove (3), one first uses for each $n>0$ the surjective map from the finite group $H^{0}(U, Y / n Y)$ onto $H^{1}(U, Y)[n]$. It shows that the finiteness of $H^{1}(U, Y)$ follows if we know that $H^{1}(U, Y)=H^{1}(U, Y)[n]$ for some $n$. Using a standard restriction-corestriction argument, this follows from the fact that

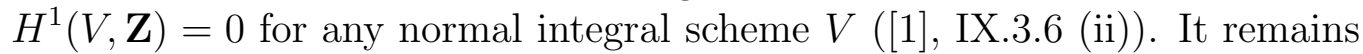
to note that the groups $H^{0}(U, A)=H^{0}(k, A)$ and $H^{0}(U, T)$ are of finite type, 
by the Mordell-Weil Theorem and Dirichlet's Unit Theorem, respectively (for the latter observe that $H^{0}(U, T)$ injects into $H^{0}(V, T) \cong\left(H^{0}\left(V, \mathbf{G}_{m}\right)\right)^{r}$, where $V / U$ is an étale covering trivialising $T)$.

Remark 3.3 The structure of the group $\mathbf{H}_{c}^{1}(U, M)$ is a bit more complicated: it is an extension of a torsion group (whose $\ell$-part is of finite cotype for all $\ell$ invertible on $U$ ) by a quotient of a profinite group.

Now, as explained on p. 159 of 9], combining a piece of the long exact sequence (8) for $\mathcal{F}^{\bullet}=\mathbf{G}_{m}$ with the main results of global class field theory yields a canonical (trace) isomorphism

$$
H_{c}^{3}\left(U, \mathbf{G}_{m}\right) \cong \mathbf{Q} / \mathbf{Z}
$$

Also, we have a natural compact support version

$$
\mathbf{H}^{i}(U, M) \times \mathbf{H}_{c}^{j}\left(U, M^{*}\right) \rightarrow \mathbf{H}_{c}^{i+j+1}\left(U, \mathbf{G}_{m}\right)
$$

of the pairing (2), constructed using the pairing (92) above. Combining the two, we get canonical pairings

$$
\mathbf{H}^{i}(U, M) \times \mathbf{H}_{c}^{2-i}\left(U, M^{*}\right) \rightarrow \mathbf{Q} / \mathbf{Z}
$$

defined for $-1 \leq i \leq 3$. For any prime number $\ell$ invertible on $U$, restricting to $\ell$-primary torsion and modding out by divisible elements (recall the notations from the beginning of the paper) induces pairings

$$
\overline{\mathbf{H}^{i}(U, M)}\{\ell\} \times \overline{\mathbf{H}_{c}^{2-i}\left(U, M^{*}\right)}\{\ell\} \rightarrow \mathbf{Q} / \mathbf{Z} .
$$

Theorem 3.4 For any 1-motive $M$ and any $\ell$ invertible on $U$, the pairing (10) is non-degenerate for $0 \leq i \leq 2$.

Note that the two groups occurring in the pairing (10) are finite by Lemma $3.2(2)$.

Proof : This is basically the argument of ([16], II.5.2 (b)). Let $n$ be a power of $\ell$. Tensoring the exact sequence

$$
0 \rightarrow \mathbf{Z} \rightarrow \mathbf{Z} \rightarrow \mathbf{Z} / n \mathbf{Z} \rightarrow 0
$$

by $M$ in the derived sense and passing to étale cohomology over $U$ induces exact sequences

$$
0 \rightarrow \mathbf{H}^{i-1}(U, M) \otimes \mathbf{Z} / n \mathbf{Z} \rightarrow \mathbf{H}^{i-1}\left(U, M \otimes^{\mathbf{L}} \mathbf{Z} / n \mathbf{Z}\right) \rightarrow \mathbf{H}^{i}(U, M)[n] \rightarrow 0
$$


Now $M \otimes^{\mathbf{L}} \mathbf{Z} / n \mathbf{Z}$ viewed as a complex of étale sheaves has trivial cohomology in degrees other than -1 ; indeed, with the notation of Section 1, the group $Y$ is torsion free and multiplication by $n$ on the group scheme $G$ is surjective in the étale topology. Therefore, using the notation of Section 1, we may rewrite the previous sequence as

$$
0 \rightarrow \mathbf{H}^{i-1}(U, M) \otimes \mathbf{Z} / n \mathbf{Z} \rightarrow H^{i}\left(U, T_{\mathbf{Z} / n \mathbf{Z}}(M)\right) \rightarrow \mathbf{H}^{i}(U, M)[n] \rightarrow 0 .
$$

Write $T(M)\{\ell\}$ for the direct limit of the groups $T_{\mathbf{Z} / n \mathbf{Z}}(M)$ as $n$ runs through powers of $\ell$ and $T_{\ell}(M)$ for their inverse limit. For each $r \geq 0, H^{r}\left(U, T_{\ell}(M)\right)$ stands for the inverse limit of $H^{r}\left(U, T_{\mathbf{Z} / n \mathbf{Z}}(M)\right.$ ) ( $n$ running through powers of $l$ ), and similarly for compact support cohomology. Passing to the direct limit in the above sequence then induces an isomorphism

$$
\overline{H^{i}(U, T(M)\{\ell\})} \cong \overline{\mathbf{H}^{i}(U, M)}\{\ell\} .
$$

Now by Artin-Verdier duality for finite sheaves ([30], 16], II.3; see also [14] in the totally imaginary case) the first group here is isomorphic (via a pairing induced by (10)) to the dual of the group $H_{c}^{3-i}\left(U, T_{\ell}\left(M^{*}\right)\right)\{\ell\}$.

Working with the analogue of exact sequence (11) for compact support cohomology and passing to the inverse limit over $n$ using the finiteness of the groups $H_{c}^{3-i}\left(U, T_{\mathbf{Z} / n \mathbf{Z}}\left(M^{*}\right)\right)$, we get isomorphisms

$$
\mathbf{H}_{c}^{2-i}\left(U, M^{*}\right)^{(\ell)}\{\ell\} \cong H_{c}^{3-i}\left(U, T_{\ell}\left(M^{*}\right)\right)\{\ell\}
$$

using the torsion freeness of the $\ell$-adic Tate module of the group $\mathbf{H}_{c}^{3-i}\left(U, M^{*}\right)$. Finally, we have $\overline{\mathbf{H}_{c}^{2-i}\left(U, M^{*}\right)}\{\ell\} \cong \mathbf{H}_{c}^{2-i}\left(U, M^{*}\right)^{(\ell)}\{\ell\}$ by the results in Lemma 3.2 and Remark 3.3

¿From now on, we shall make the convention (to ease notation) that for any archimedean place $v$ and each $i \in \mathbf{Z}, \mathbf{H}^{i}\left(k_{v}, M\right)$ means the modified group $\widehat{\mathbf{H}}^{i}\left(k_{v}, M\right)$ (In particular it is zero if $v$ is complex, and it is a finite 2-torsion group if $v$ is real).

Following [16], II.5, we define for $i \geq 0$

$$
D^{i}(U, M)=\operatorname{Ker}\left[\mathbf{H}^{i}(U, M) \rightarrow \prod_{v \in \Sigma} \mathbf{H}^{i}\left(k_{v}, M\right)\right]
$$

where the finite subset $\Sigma=\Sigma_{f} \cup \Omega_{\infty} \subset \Omega_{k}$ consists of the real places and the primes of $\mathcal{O}_{k}$ which do not correspond to a closed point of $U$. For $i \geq 0$ we also have

$$
D^{i}(U, M)=\operatorname{Im}\left[\mathbf{H}_{c}^{i}(U, M) \rightarrow \mathbf{H}^{i}(U, M)\right]
$$


by the definition of compact support cohomology. By Lemma 3.2, $D^{1}(U, M)$ is a torsion group and $D^{1}(U, M)\{\ell\}$ is of finite cotype. Now Theorem 3.4 has the following consequence:

Corollary 3.5 Under the notation and assumptions of Theorem 3.4. for $i=0,1$ there is a pairing

$$
D^{i}(U, M)\{\ell\} \times D^{2-i}\left(U, M^{*}\right)\{\ell\} \rightarrow \mathbf{Q} / \mathbf{Z}
$$

whose left and right kernels are respectively the divisible subgroups of the two groups.

\section{Remarks 3.6}

1. As $D^{0}(U, M)$ is of finite type, the divisible part of $D^{0}(U, M)\{\ell\}$ is zero.

2. In the case $M=[0 \rightarrow T]$, the corollary gives back (part of) [16], Corollary II.4.7, itself based on the main result of Deninger [4] (which we do not use here).

Proof : The proof works along the same pattern as that used in the case $i=1, M=[0 \rightarrow A]$ treated in [16], Corollary II.5.3. As the proof given in loc. cit. contains some inaccuracies, we give a detailed argument. We use the following commutative diagram whose exact rows come from the definition of the groups $D^{i}$ and whose vertical maps are induced by the pairings (10) and (5).

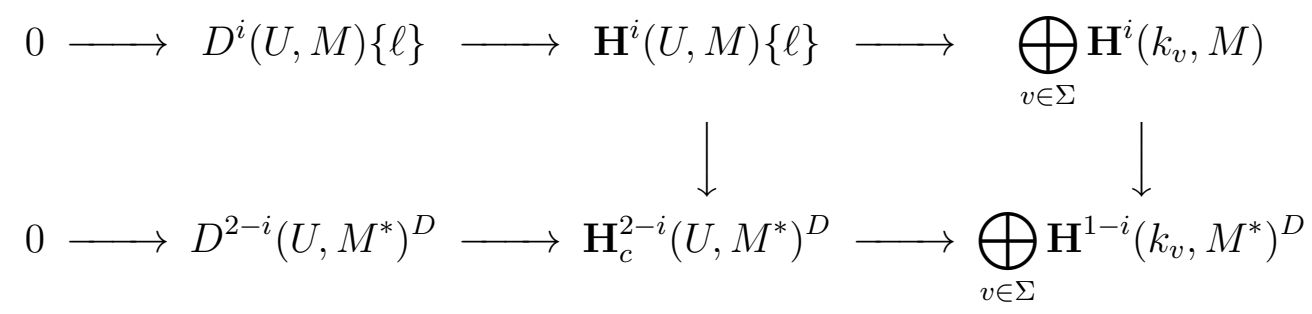

The diagram defines a map $D^{i}(U, M)\{\ell\} \rightarrow D^{2-i}\left(U, M^{*}\right)^{D}$. The right vertical map is injective for $i=0$ by Theorem 2.3, Remark 2.4, the first statement in Proposition 2.8, and Proposition 2.9. For $i=1$ it is injective by Proposition 2.9 and the second statement in Proposition 2.8 (indeed for $v$ finite, $\mathbf{H}^{0}\left(k_{v}, M^{*}\right)$ is now equipped with the discrete topology, which is finer than the topology defined in Proposition 2.8). The middle vertical map has divisible kernel by Theorem 3.4. Hence the map $D^{i}(U, M)\{\ell\} \rightarrow D^{2-i}\left(U, M^{*}\right)^{D}$ has divisible kernel, and similarly for the induced map $D^{i}(U, M)\{\ell\} \rightarrow$ 
$D^{2-i}\left(U, M^{*}\right)\{\ell\}^{D}$ (recall that $D^{1}(U, M)$ and $D^{2}(U, M)$ are torsion). Now exchange the roles of $M$ and $M^{*}$.

Let $A_{k}$ denote the generic fibre of $A$ and

$$
\amalg^{1}\left(A_{k}\right):=\operatorname{Ker}\left[H^{1}\left(k, A_{k}\right) \rightarrow \prod_{v \in \Omega_{k}} H^{1}\left(\hat{k}_{v}, A_{k}\right)\right]
$$

its Tate-Shafarevich group. A special case of Corollary 3.5 is

Proposition 3.7 Let $M$ be a 1-motive over $U$ and $\ell$ a prime number invertible on $U$. Assume that $\amalg^{1}\left(A_{k}\right)\{\ell\}$ and $\amalg^{1}\left(A_{k}^{*}\right)\{\ell\}$ are finite. Then the pairing

$$
D^{1}(U, M)\{\ell\} \times D^{1}\left(U, M^{*}\right)\{\ell\} \rightarrow \mathbf{Q} / \mathbf{Z}
$$

of Corollary 3.5 is a perfect pairing of finite groups.

Proof : Using Corollary 3.5, it is sufficient to prove that $D^{1}(U, M)\{\ell\}$ is finite. We have a commutative diagram with exact rows:

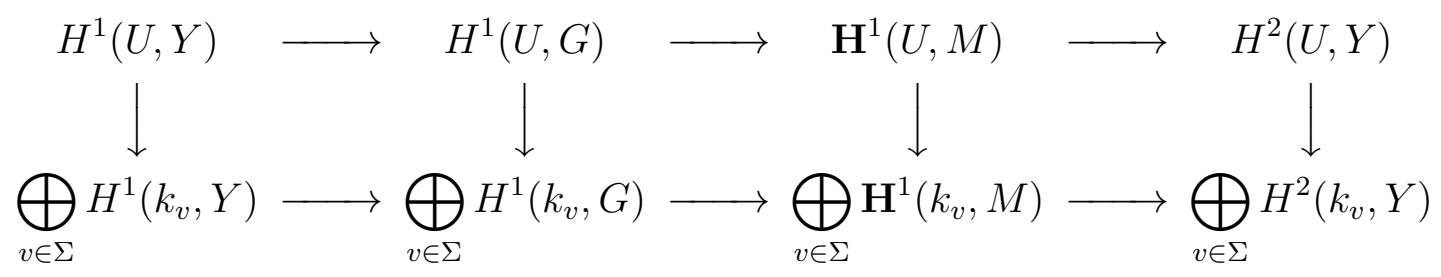

An inspection of the diagram reveals that for the finiteness of $D^{1}(U, M)\{\ell\}$ it suffices to show (using finiteness of $\Sigma$ ) the finiteness of the torsion groups $D^{1}(U, G)\{\ell\}, H^{1}\left(k_{v}, Y\right)$ and $D^{2}(U, Y)$, respectively.

For the first, note that the assumption on $\amalg^{1}\left(A_{k}\right)$ implies that $D^{1}(U, A)\{\ell\}$ is finite by [16, II.5.5, whence the required finiteness follows from the finiteness of $H^{1}(U, T)$ ([16], II.4.6). We have seen the second finiteness several times when discussing local duality. The finiteness of $D^{2}(U, Y)$ follows from that of $H_{c}^{2}(U, Y)$ which is is dual to the finite group $H^{1}(U, T)$ by a result of Deninger ([16], II.4.6). (One can also prove the finiteness of $D^{2}(U, Y)$ by the following more direct reasoning: using a restriction-corestriction argument, one reduces to the case $Y=\mathbf{Z}$. Then $D^{2}(U, \mathbf{Z})=D^{1}(U, \mathbf{Q} / \mathbf{Z})$ is the dual of the Galois group of the maximal abelian extension of $k$ unramified over $U$ and totally split at the places outside $U$, and hence is finite by global class field theory.)

Remark 3.8 The same argument shows that the finiteness of $\amalg^{1}\left(A_{k}\right)$ implies the finiteness of $D^{1}(U, M)$. 


\section{Global results: relation to Galois cohomol- ogy}

The notation and assumptions are the same as in the previous section. In particular $U$ is an affine open subset of $\operatorname{Spec} \mathcal{O}_{k}$ and $\Sigma \subset \Omega_{k}$ consists of the real places and the finite places of $\operatorname{Spec} \mathcal{O}_{k} \backslash U$. Fix an algebraic closure $\bar{k}$ of $k$ (corresponding to a geometric point $\bar{\eta}$ of $U$ ). Let $\Gamma_{\Sigma}=\pi_{1}(U, \bar{\eta})$ be the Galois group of the maximal subfield $k_{\Sigma}$ of $\bar{k}$ such that the extension $k_{\Sigma} / k$ is unramified outside $\Sigma$. When $G_{k}$ is the restriction of a $U$-group scheme $G$ to Spec $k$, we shall write $H^{i}\left(\Gamma_{\Sigma}, G_{k}\right)$ for $H^{i}\left(\Gamma_{\Sigma}, G_{k}\left(k_{\Sigma}\right)\right)$.

We begin with the following analogue of [16], II.2.9:

Proposition 4.1 Let $M=[Y \rightarrow G]$ be a 1-motive over $U$ and $M_{k}$ its restriction to Spec $k$. Let $\ell$ be a prime number invertible on $U$. Then:

1. The natural map $\mathbf{H}^{i}(U, M)\{\ell\} \rightarrow \mathbf{H}^{i}\left(\Gamma_{\Sigma}, M_{k}\right)\{\ell\}$ is an isomorphism for $i \geq 1$.

2. The natural map $\mathbf{H}^{1}(U, M)\{\ell\} \rightarrow \mathbf{H}^{1}\left(k, M_{k}\right)\{\ell\}$ is injective.

3. The natural map $\mathbf{H}^{0}(U, M) \rightarrow \mathbf{H}^{0}\left(k, M_{k}\right)$ is injective.

Proof : $\quad$ To prove (1) in the case $i>1$, pass to the direct limit over powers of $\ell$ in exact sequence (11). Then Lemma 3.2 (1) implies that it is sufficient to prove the statement for $M$ replaced by $T(M)\{\ell\}$, which follows from [16], II.2.9 by passing to the limit. In the case $i=1$ one unscrews $M$ using the weight filtration and applies [16], II.2.9, II.4.14 and II.5.5., using also the fact that $A(k)=H^{0}(U, A)=H^{0}\left(\Gamma_{\Sigma}, A_{k}\right)$ for the abelian scheme $A$ with generic fibre $A_{k}$.

For (2), it is sufficient by (1) to show that the canonical map $\mathbf{H}^{1}\left(\Gamma_{\Sigma}, M_{k}\right) \rightarrow$ $\mathbf{H}^{1}\left(k, M_{k}\right)$ is injective. Again using the weight filtration on $M$, one reduces this to the injectivity of $\mathbf{H}^{1}\left(\Gamma_{\Sigma}, G_{k}\right) \rightarrow \mathbf{H}^{1}\left(k, G_{k}\right)$, the injectivity of $H^{2}\left(\Gamma_{\Sigma}, Y_{k}\right) \rightarrow H^{2}\left(k, Y_{k}\right)$ and the surjectivity of $H^{1}\left(\Gamma_{\Sigma}, Y_{k}\right) \rightarrow H^{1}\left(k, Y_{k}\right)$ (Note that $Y_{k}(\bar{k})=Y_{k}\left(k_{\Sigma}\right)$ because there exists a finite étale covering $\widetilde{U} / U$ such that $Y \times_{U} \widetilde{U}$ is constant). The two injectivities are consequences of the restriction-inflation sequence for $H^{1}$ in Galois cohomology, noting the isomorphisms

$H^{2}\left(\Gamma_{\Sigma}, Y_{k}\right) \cong H^{1}\left(\Gamma_{\Sigma}, Y_{k}(\bar{k}) \otimes \mathbf{Q} / \mathbf{Z}\right) \quad$ and $\quad H^{2}\left(k, Y_{k}\right) \cong H^{1}\left(k, Y_{k}(\bar{k}) \otimes \mathbf{Q} / \mathbf{Z}\right)$ 
for the lattice $Y_{k}(\bar{k})=Y_{k}\left(k_{\Sigma}\right)$. The surjectivity follows from the triviality of the abelian group $H^{1}\left(\operatorname{Gal}\left(\bar{k} / k_{\Sigma}\right), Y_{k}(\bar{k})\right)=\operatorname{Hom}_{\text {cont }}\left(\operatorname{Gal}\left(\bar{k} / k_{\Sigma}\right), Y_{k}(\bar{k})\right)$, the Galois group Gal $\left(\bar{k} / k_{\Sigma}\right)$ being profinite and $Y_{k}(\bar{k})$ torsion-free.

The statement (3) is obvious for $M=[0 \rightarrow G]$ (a morphism from $U$ to $G$ is trivial if and only if it is trivial at the generic point). Using the weight filtration, it is sufficient to prove that the map $H^{0}(U, Y) \rightarrow H^{0}\left(k, Y_{k}\right)$ is surjective and the map $H^{1}(U, Y) \rightarrow H^{1}\left(k, Y_{k}\right)$ is injective. Actually $H^{0}(U, Y)=H^{0}\left(k, Y_{k}\right)$ and $H^{0}(U, Y / n Y)=H^{0}\left(k, Y_{k} / n Y_{k}\right)$ for each $n>0$ because $Y$ and $Y / n Y$ are locally constant in the étale topology. The injectivity of the map $H^{1}(U, Y) \rightarrow H^{1}\left(k, Y_{k}\right)$ now follows from the commutative exact diagram

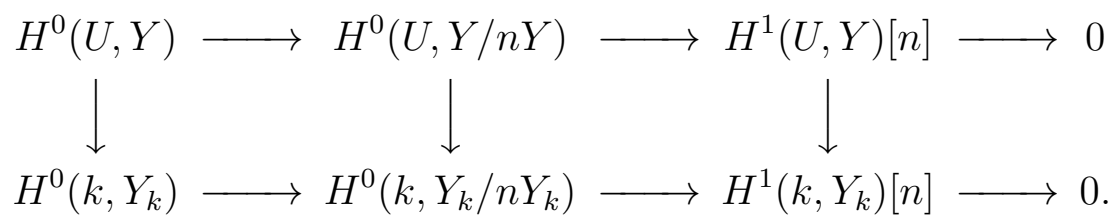

For a 1-motive $M_{k}$ over $k$ and $i \geq 0$, define the Tate-Shafarevich groups

$$
\amalg^{i}\left(M_{k}\right)=\operatorname{Ker}\left[\mathbf{H}^{i}\left(k, M_{k}\right) \rightarrow \prod_{v \in \Omega_{k}} \mathbf{H}^{i}\left(\hat{k}_{v}, M_{k}\right)\right]
$$

If $M_{k}$ is the restriction of a 1-motive $M$ defined over $U$, we also define

$$
\amalg_{\Sigma}^{i}\left(M_{k}\right):=\operatorname{Ker}\left[\mathbf{H}^{i}\left(\Gamma_{\Sigma}, M_{k}\right) \rightarrow \prod_{v \in \Sigma} \mathbf{H}^{i}\left(\hat{k}_{v}, M_{k}\right)\right]
$$

Remark 4.2 By Lemma 2.7, we can replace $\hat{k}_{v}$ by $k_{v}$ in the definition of $\amalg^{i}$ for $i \geq 0$.

Proposition 4.1. Remark 4.2 and Corollary 3.5 imply immediately:

Corollary 4.3 Let $M$ be a 1-motive over $U$ and $\ell$ a prime number invertible on $U$. Then the pairing (12) can be identified with a pairing

$$
\amalg_{\Sigma}^{1}\left(M_{k}\right)\{\ell\} \times \amalg_{\Sigma}^{1}\left(M_{k}^{*}\right)\{\ell\} \rightarrow \mathbf{Q} / \mathbf{Z},
$$

nondegenerate modulo divisible subgroups.

In the remaining of this section, we prove Theorem 0.2. As in ([16, II.6), the idea is to identify the Tate-Shafarevich groups with the groups $D^{i}(U, M)$ considered in the previous section for $U$ sufficiently small. But a difficulty is that when $Y$ is not trivial, the restriction of $D^{i}(U, M)$ to $\mathbf{H}^{i}(V, M)$ for $V \subset U$ need not be a subset of $D^{i}(V, M)$, as the following example shows. 
Example 4.4 Let $k$ be a totally imaginary number field with $\operatorname{Pic} \mathcal{O}_{k} \neq 0$ and $M:=\mathbf{Z}[1]$. Then for $U=\operatorname{Spec} \mathcal{O}_{k}$ we have $D^{1}(U, M)=H^{1}(U, \mathbf{Q} / \mathbf{Z}) \neq 0$ by global class field theory. Let $\alpha \neq 0$ in $H^{1}(U, \mathbf{Q} / \mathbf{Z})$. Then there is a finite place $v$ coming from a closed point of $U$ such that the restriction of $\alpha$ to $H^{1}\left(k_{v}, \mathbf{Q} / \mathbf{Z}\right)$ is nonzero, for otherwise we would get $\alpha=0$ by Chebotarev's density theorem. Therefore the restriction of $\alpha$ to $H^{1}(V, M)$ does not belong to $D^{1}(V, M)$ for $V=U-\{v\}$.

Thus the identification of $\amalg^{1}\left(M_{k}\right)$ with some $D^{1}(U, M)$ for suitable $U$ is not straightforward, in contrast to the case of abelian varieties. The following proposition takes care of this problem.

Note that by Proposition 4.1, any $D^{1}(U, M)\{\ell\}$ is naturally a subgroup of $H^{1}\left(k, M_{k}\right)$ for $\ell$ invertible on $U$.

Proposition 4.5 Let $M=[Y \rightarrow G]$ be a 1-motive over $U$ and $\ell$ a prime invertible on $U$. There exists an open subset $U_{0} \subset U$ such that for any open subset $U_{1} \subset U_{0}$, the group $D^{1}\left(U_{1}, M\right)\{\ell\}$ as a subgroup of $H^{1}\left(k, M_{k}\right)$ is contained in $\amalg^{1}\left(M_{k}\right)\{\ell\}$.

For the proof of this proposition we need two preliminary lemmas.

Lemma 4.6 Let $v$ be a finite place of $U$ and $\mathcal{O}_{v}$ the henselisation of the local ring of $U$ at $v$. Then the natural map $H^{2}\left(\mathcal{O}_{v}, Y\right) \rightarrow H^{2}\left(k_{v}, Y\right)$ is injective when restricted to $\ell$-primary torsion.

Note that the groups occurring in the lemma are torsion groups (for the first one, this follows from Lemma 3.2 (1) applied to $M=Y[1]$ and passing to the limit).

Proof : By the localisation sequence for the pair $\operatorname{Spec} k_{v} \subset \operatorname{Spec} \mathcal{O}_{v}$ it is enough to prove triviality of the group $H_{v}^{2}\left(\mathcal{O}_{v}, Y\right)\left[\ell^{n}\right]$ for any $n$. This group is a quotient of $H_{v}^{1}\left(\mathcal{O}_{v}, Y / \ell^{n} Y\right)$ which, according to [16], II.1.10 (a), is a finite group dual to $H^{2}\left(\mathcal{O}_{v}, \underline{\operatorname{Hom}}\left(Y / \ell^{n} Y, \mathbf{G}_{m}\right)\right)$. But for any finite sheaf $F$ over $\mathcal{O}_{v}$ of order prime to the characteristic of the residue field $\kappa(v)$ of $v$, we have $H^{2}\left(\mathcal{O}_{v}, F\right)=0$ because $H^{2}\left(\mathcal{O}_{v}, F\right)=H^{2}(\kappa(v), \widetilde{F})$ by [15], III.3.11 a) (where $\widetilde{F}$ is the restriction of $F$ to $\operatorname{Spec} \kappa(v)$ ), and $\kappa(v)$ is of cohomological dimension 1 .

Lemma 4.7 There exists an open subset $U_{0} \subset U$ such that for any open subset $U_{1} \subset U_{0}$, the group $\amalg^{2}\left(Y_{k}\right)\{\ell\}$ contains $D^{2}\left(U_{1}, Y\right)\{\ell\}$.

Note that according to Proposition 4.1, for any open $V \subset U$ the group $D^{2}(V, Y)\{\ell\}=D^{1}(V, Y[1])\{\ell\}$ identifies with a subgroup of $H^{2}(k, Y)$. 
Proof : By definition we have $\amalg^{2}\left(Y_{k}\right)\{\ell\} \supset \bigcap_{V \subset U} D^{2}(V, Y)\{\ell\}$. The group $D^{2}(V, Y)$ is finite for each open subset $V \subset U$ (cf. proof of Proposition 3.7). Therefore there exist finitely many open subsets $V_{1}, \ldots, V_{r}$ of $U$ such that $\amalg^{2}\left(Y_{k}\right)\{\ell\} \supset \bigcap_{i=1}^{r} D^{2}\left(V_{i}, Y\right)\{\ell\}$. Let $U_{0}$ be the intersection $\bigcap_{i=1}^{r} V_{i}$. As the groups $H_{c}^{2}(-,-Y)$ are covariantly functorial for open immersions $U_{1} \subset U_{2}$ (see the previous section), for $i=1, \ldots, r$ there are natural maps $H_{c}^{2}\left(U_{0}, Y\right) \rightarrow$ $H_{c}^{2}\left(V_{i}, Y\right) \rightarrow H^{2}(k, Y)$ which factor through $D^{2}\left(U_{0}, Y\right)$, so that we get inclusions $D^{2}\left(U_{0}, Y\right) \hookrightarrow D^{2}\left(V_{i}, Y\right)$ and finally $D^{2}\left(U_{0}, Y\right)\{\ell\} \hookrightarrow \amalg^{2}(k, Y)\{\ell\}$. The above of course holds for any $U_{1} \subset U_{0}$ instead of $U_{0}$.

Proof of Proposition 4.5: Choose $U_{0}$ as in the previous lemma and take $U_{1} \subset U_{0}$. It suffices to show that for each closed point $v \in U_{1}$ the group $D^{1}\left(U_{1}, M\right)\{\ell\}$ maps to 0 by the restriction map $\mathbf{H}^{1}\left(U_{1}, M\right) \rightarrow \mathbf{H}^{1}\left(k_{v}, M\right)$. Now there is a commutative diagram with exact rows

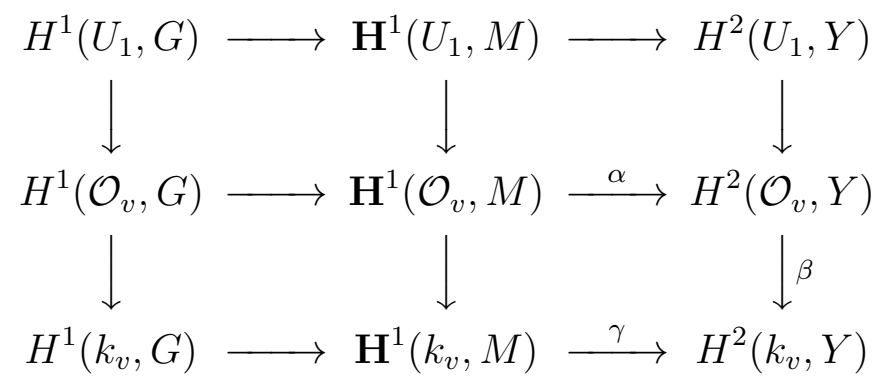

Here the group $H^{1}\left(\mathcal{O}_{v}, G\right)$ is zero because $G$ is smooth and connected over $U_{1}$, hence $H^{1}\left(\mathcal{O}_{v}, G\right)=H^{1}\left(\mathbf{F}_{v}, \widetilde{G}\right)$ (cf. [15], III.3.11 a)) is trivial by Lang's Theorem. So the map $\alpha$ in the diagram is injective, but when restricted to $\ell$ primary torsion, the map $\beta$ is injective as well, by Lemma 4.6. Therefore the image of the map $D^{1}\left(U_{1}, M\right)\{\ell\} \rightarrow \mathbf{H}^{1}\left(k_{v}, M\right)$ injects into $H^{2}\left(k_{v}, Y\right)$ by $\gamma$, so we reduce to the triviality of the composite map $D^{1}\left(U_{1}, M\right)\{\ell\} \rightarrow H^{2}\left(k_{v}, Y\right)$. This in turn follows from Lemma 4.7 as the map factors through $D^{2}\left(U_{1}, Y\right)\{\ell\}$ by the right half of the diagram.

We are now able to prove one half of Theorem 0.2 ,

Theorem 4.8 Let $M_{k}$ be a 1-motive over $k$. Then there exists a canonical pairing

$$
\amalg^{1}\left(M_{k}\right) \times \amalg^{1}\left(M_{k}^{*}\right) \rightarrow \mathbf{Q} / \mathbf{Z}
$$

whose kernels are the maximal divisible subgroups of each group. 
Proof : We construct the pairing separately for each prime $\ell$. Let $U$ be an open subset of $\operatorname{Spec} \mathcal{O}_{k}$ such that $M_{k}$ is the restriction of a 1-motive $M$ defined over $U$ and $\ell$ is invertible on $U$. Take a subset $U_{0}$ as in Proposition 4.5. We contend that the inclusion $D^{1}\left(U_{0}, M\right)\{\ell\} \subset \amalg^{1}\left(M_{k}\right)\{\ell\}$ furnished by the proposition is in fact an equality, from which the theorem will follow by Corollary 3.5. Indeed, any element of $\amalg^{1}\left(M_{k}\right)\{\ell\}$ is contained in some $D^{1}(V, M)\{\ell\}$, where we may assume $V \subset U_{0}$. But $D^{1}(V, M)\{\ell\} \subset D^{1}\left(U_{0}, M\right)\{\ell\} \subset \amalg^{1}\left(M_{k}\right)$, by the same argument as in the end of the proof of Lemma 4.7 .

Corollary 4.9 Let $M_{k}$ be a 1-motive over $k$. If $\amalg^{1}\left(A_{k}\right)$ and $\amalg^{1}\left(A_{k}^{*}\right)$ are finite, then there is a perfect pairing of finite groups

$$
\amalg^{1}\left(M_{k}\right) \times \amalg^{1}\left(M_{k}^{*}\right) \rightarrow \mathbf{Q} / \mathbf{Z}
$$

Proof : Apply Theorem 4.8, Proposition [3.7, and Remark 3.8

Remark 4.10 In the case $M_{k}=\left[0 \rightarrow T_{k}\right]$, we recover the duality between $\amalg^{1}\left(T_{k}\right)$ and $\amalg^{2}\left(Y_{k}^{*}\right)$, where $Y_{k}^{*}$ is the module of characters of the torus $T_{k}$. See [27] and [18], VIII.6.8.

Now to the result for $\amalg^{0}$ and $\amalg^{2}$. First we show:

Lemma 4.11 Let $M_{k}$ be a 1-motive over $k$. Then $\amalg^{0}\left(M_{k}\right)$ is finite.

Proof : We prove that the map $\mathbf{H}^{0}\left(k, M_{k}\right) \rightarrow \mathbf{H}^{0}\left(\hat{k}_{v}, M_{k}\right)$ has finite kernel for any finite place $v$ of $k$. With the same notation as above, there is a commutative diagram with exact rows:

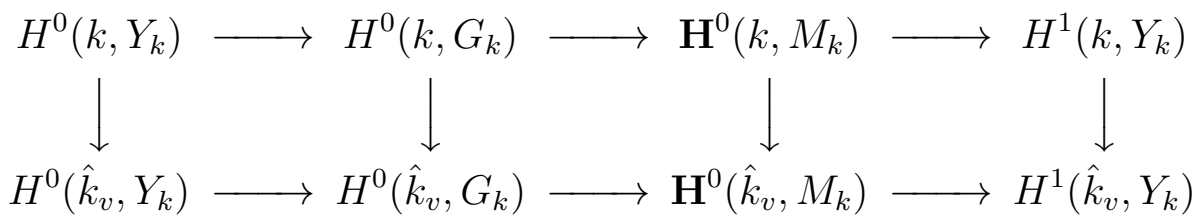

The group $H^{1}\left(k, Y_{k}\right)$ is finite because $Y_{k}(\bar{k})$ is a lattice. This being said, a diagram chase using the injectivity of the second vertical map reveals that it suffices to establish the finiteness of the cokernel of the first vertical map. Now this cokernel is certainly of finite type (as $H^{0}\left(\hat{k}_{v}, Y_{k}\right)$ is), and it is annihilated by the degree of a finite field extension trivialising the Galois action on $Y_{k}(\bar{k})$, by a restriction-corestriction argument. 
Proposition 4.12 Let $M_{k}$ be a 1-motive over $k$. Then there is a canonical pairing

$$
\amalg^{0}\left(M_{k}\right) \times \amalg^{2}\left(M_{k}^{*}\right) \rightarrow \mathbf{Q} / \mathbf{Z}
$$

whose left kernel is trivial and right kernel is the maximal divisible subgroup of $\amalg^{2}\left(M_{k}^{*}\right)$.

Proof : By Lemma 4.11 it is sufficient to construct (for each prime number $\ell$ ) a pairing $\amalg^{0}\left(M_{k}\right)\{\ell\} \times \amalg^{2}\left(M_{k}^{*}\right)\{\ell\} \rightarrow \mathbf{Q} / \mathbf{Z}$ with the required properties. Let $U$ be an open subset of $\operatorname{Spec} \mathcal{O}_{k}$ such that $\ell$ is invertible on $U$ and $M$ extends to a 1-motive $M$ over $U$. Since $D^{0}(V, M)\{\ell\}$ is finite for each open subset $V$ of $U$ by lemma 3.2 (3), the same argument as in Lemma 4.7 shows that there exists $U_{0} \subset U$ such that $\amalg^{0}\left(M_{k}\right)\{\ell\}=D^{0}(V, M)\{\ell\}$ for any open subset $V$ of $U_{0}$ (recall that $D^{0}(V, M)$ injects into $\mathbf{H}^{0}\left(k, M_{k}\right)$ by Lemma 4.1, (3)).

Now notice that $\mathbf{H}^{2}\left(\mathcal{O}_{v}, M\right)=0$ for each finite place $v$ of $U$. Indeed, we have $\mathbf{H}^{2}\left(\mathcal{O}_{v}, M\right)=\mathbf{H}^{2}\left(\mathbf{F}_{v}, \widetilde{M}\right)$ by [15], III.3.11, and we have seen in the proof of Theorem 2.10 that $H^{2}\left(\mathbf{F}_{v}, \widetilde{G}\right)=0$ and $H^{3}\left(\mathbf{F}_{v}, \widetilde{Y}\right)$ is zero because $\mathbf{F}_{v}$ is of strict cohomological dimension 2, whence the claim by dévissage. It follows that for each open subset $V$ of $U$, the image of the restriction map $D^{2}(U, M) \rightarrow \mathbf{H}^{2}(V, M)$ lies in $D^{2}(V, M)$. Using the covariant functoriality of compact support cohomology for open immersions, we also have $\mathcal{D}^{2}(V, M) \subset$ $\mathcal{D}^{2}(U, M)$, and finally $\mathcal{D}^{2}(V, M)=\mathcal{D}^{2}(U, M)$, where $\mathcal{D}^{2}(-, M)$ stands for the image of $D^{2}(\ldots, M)$ in $\mathbf{H}^{2}\left(k, M_{k}\right)$. In particular, $\amalg^{2}\left(M_{k}\right)=\mathcal{D}^{2}(V, M)$ for each open subset $V$ of $U_{0}$. On the other hand the map $D^{2}(V, M)\{\ell\} \rightarrow$ $\mathbf{H}^{2}\left(\Gamma_{\Sigma_{V}}, M_{k}\right)$ is injective, where $\Sigma_{V}$ consists of the places of $k$ which are not in $V$ (Proposition 4.1, (1)). Therefore the natural map

$$
\lim _{\longrightarrow} D^{2}(V, M)\{\ell\} \rightarrow \amalg^{2}\left(M_{k}\right)\{\ell\}
$$

(here the limit is taken over nonempty open subsets $V$ of $U_{0}$ ) is an isomorphism. The proposition follows from the identification of $D^{0}(V, M)\{\ell\}$ with $\amalg^{0}\left(M_{k}\right)\{\ell\}$ and from the case $i=0$ in Corollary 3.5 .

\section{Remarks 4.13}

1. The special case $M_{k}=Y_{k}[1]$ corresponds to the duality between the groups $\amalg^{1}\left(Y_{k}\right)$ and $\amalg^{2}\left(T_{k}^{*}\right)$, where $T_{k}^{*}$ is the torus with character module $Y_{k}$. Compare [16], I.4.20 (a). 
2. The case $M_{k}=\left[0 \rightarrow A_{k}\right]$ does not contain much new information. Indeed, assume for instance $k$ totally imaginary. Then combining the injectivity of $A_{k}(k) \rightarrow A_{k}\left(\hat{k}_{v}\right)$ as well as the vanishing of $H^{2}\left(\hat{k}_{v}, A_{k}\right)$ for each finite place $v([23]$, II.5.3, Proposition 16) with the above proposition we get that $H^{2}\left(k, A_{k}\right)$ is a divisible group; but this we know anyway since $k$ is of cohomological dimension 2. In fact in this case $H^{2}\left(k, A_{k}\right)=0$ by [16], I.6.24.

3. Presumably, $\amalg^{2}(M)$ is finite, as it is finite for $M=T$ and even trivial for $M=A$ and $M=Y[1]$.

\section{The Poitou-Tate exact sequence}

We keep notation from the previous sections. In particular, $k$ is a number field, $M_{k}$ is a 1-motive over $k$, and $U$ is an open subset of $\operatorname{Spec} \mathcal{O}_{k}$ such that $M_{k}$ extends to a 1-motive $M$ over $U$. For each finite place $v$ of $k$, we denote by $\widehat{\mathcal{O}}_{v}$ the ring of integers of the completion $\hat{k}_{v}$. The groups $\mathbf{H}^{i}\left(k, M_{k}\right)$ $(-1 \leq i \leq 2)$ and $\mathbf{H}^{i}\left(\hat{k}_{v}, M_{k}\right)$ for $i \geq 1\left(v \in \Omega_{k}\right)$ are equipped with the discrete topology. For $i \geq 0$, we define $\mathbf{P}^{i}\left(M_{k}\right)$ as the restricted product over $v \in \Omega_{k}$ of the $\mathbf{H}^{i}\left(\hat{k}_{v}, M_{k}\right)$, with respect to the images of $\mathbf{H}^{i}\left(\widehat{\mathcal{O}}_{v}, M\right)$ for finite places $v$ of $U$. The groups $\mathbf{P}^{i}\left(F_{k}\right)$ are defined similarly for any étale sheaf $F_{k}$ over Spec $k$. Clearly all these groups are independent of the choice of $U$. We equip them with their restricted product topology. Note that $\mathbf{P}^{2}\left(M_{k}\right)$ is the direct sum of the groups $\mathbf{H}^{2}\left(\hat{k}_{v}, M_{k}\right)$ for all places $v$ : indeed, for each finite place $v$ of $U$, we have $\mathbf{H}^{2}\left(\hat{\mathcal{O}}_{v}, M\right)=\mathbf{H}^{2}\left(\mathbf{F}_{v}, \widetilde{M}\right)$, and we have already seen that this last group vanishes. We have natural restriction maps $\beta_{i}: \mathbf{H}^{i}\left(k, M_{k}\right) \rightarrow \mathbf{P}^{i}\left(M_{k}\right)$ for $i=1,2$; their kernels are precisely the groups $\amalg^{i}\left(M_{k}\right)$.

In this section we establish a Poitou-Tate type exact sequence for $M_{k}$. A technical complication for our considerations to come arises from the fact that one is forced to work with two kinds of completions: the first one is what we have used up till now, i.e. the inverse limit $A^{\wedge}$ of all open subgroups of finite index of a topological abelian group $A$, and the second is the inverse limit $A_{\wedge}$ of the quotients $A / n$ for all $n>0$. These are not the same in general (indeed, the latter is not necessarily profinite). But it is easy to see that $\left(A_{\wedge}\right)^{\wedge}$ is naturally isomorphic to $A^{\wedge}$; in particular, we have a natural $\operatorname{map} A_{\wedge} \rightarrow A^{\wedge}$.

The natural map $\mathbf{H}^{0}(k, M) \rightarrow \mathbf{P}^{0}(k, M)$ therefore gives rise to a commu- 
tative diagram between the two different kinds of completions:

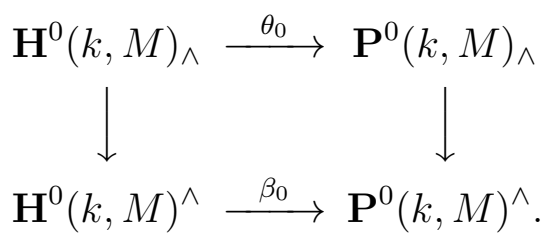

Denote by $\amalg_{\wedge}^{0}\left(M_{k}\right)$ the kernel of the above map $\theta_{0}$. We first prove the following duality result analogous to [16, I.6.13 (b).

Proposition 5.1 Let $M_{k}=\left[Y_{k} \rightarrow G_{k}\right]$ be a 1-motive over $k$. Assume that $\amalg^{1}\left(A_{k}\right)$ is finite. Then there is a perfect pairing

$$
\amalg_{\wedge}^{0}\left(M_{k}\right) \times \amalg^{2}\left(M_{k}^{*}\right) \rightarrow \mathbf{Q} / \mathbf{Z}
$$

where the first group is compact and the second is discrete.

\section{Remarks 5.2}

1. The proof below will show that if one only assumes the finiteness of the $\ell$-primary torsion part of $\amalg^{1}\left(A_{k}\right)$, then one gets a similar duality between the $\ell$-primary torsion part of $\amalg^{2}\left(M_{k}^{*}\right)$ and a group $\amalg_{(\ell)}^{0}\left(M_{k}\right)$ defined similarly to $\amalg_{\wedge}^{0}\left(M_{k}\right)$ but taking only $\ell$-adic completions.

2. It is natural to ask how the above proposition is related to Proposition 4.12. In the case when $M_{k}^{*}=\left[0 \rightarrow T_{k}\right]$ is a torus, one knows that $\amalg^{2}\left(M_{k}^{*}\right)=\amalg^{2}\left(T_{k}\right)$ is finite and so by comparing the two statements we get that $\amalg_{\wedge}^{0}\left(M_{k}\right)=\amalg^{0}\left(M_{k}\right)$ and the two dualities are the same. In the case $M_{k}=\left[0 \rightarrow A_{k}\right]$, both $\amalg_{\wedge}^{0}\left(A_{k}\right)$ and $\amalg^{2}\left(A_{k}\right)$ are trivial (16], Corollaries I.6.23 and I.6.24), but even the fact that they are dual groups does not seem to be an easy consequence of Proposition 4.12. The equality $\amalg^{0}(M)=\amalg_{\wedge}^{0}(M)$ would follow via Proposition 4.12 from the finiteness of $\amalg^{2}\left(M^{*}\right)$, but we do not know this latter fact (compare Remark $4.13(3)$ ).

For the proof of the proposition we first show a lemma.

Lemma 5.3 For any $n>0$, the natural map

$$
\mathbf{P}^{0}\left(M_{k}\right) / n \rightarrow \prod_{v} \mathbf{H}^{0}\left(\hat{k}_{v}, M_{k}\right) / n
$$

is injective and its image is the restricted product of the groups $\mathbf{H}^{0}\left(\hat{k}_{v}, M_{k}\right) / n$ with respect to the subgroups $\mathbf{H}^{0}\left(\hat{\mathcal{O}}_{v}, M_{k}\right) / n$. 
Proof : Take an element $x=\left(x_{v}\right) \in \mathbf{P}^{0}\left(M_{k}\right)$ and assume $x_{v}$ is in $n \mathbf{H}^{0}\left(\hat{k}_{v}, M\right)$ for all $v$. For almost all $v$, it also comes from $\mathbf{H}^{0}\left(\hat{\mathcal{O}}_{v}, M\right)$, hence in fact it comes from $n \mathbf{H}^{0}\left(\hat{\mathcal{O}}_{v}, M\right)$, for in the exact commutative diagram

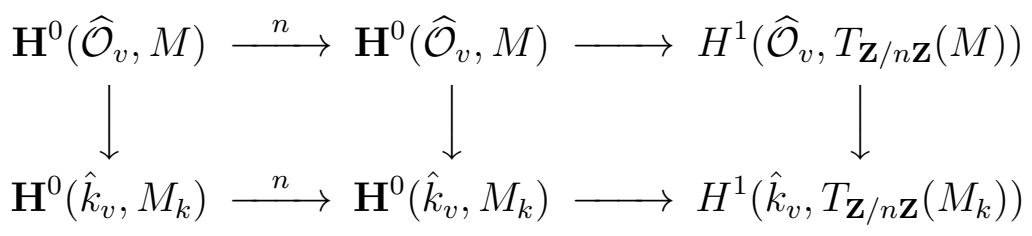

the third vertical map is injective, $H_{v}^{1}\left(\widehat{\mathcal{O}}_{v}, T_{\mathbf{Z} / n \mathbf{Z}}(M)\right)$ being trivial by the same argument as in the proof of Lemma 4.6] But this means $x \in n \mathbf{P}^{0}\left(M_{k}\right)$. The second statement is obvious.

Proof of Proposition 5.1; Using exact sequence (11) we get a commutative diagram with exact rows for each $n>0$ :

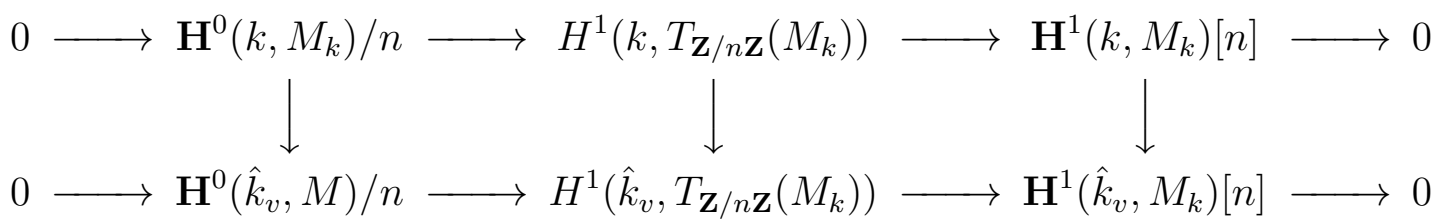

and a similar exact diagram holds with $\widehat{\mathcal{O}}_{v}$ instead of $\hat{k}_{v}$. Taking restricted products and using the above lemma, we get a commutative exact diagram:

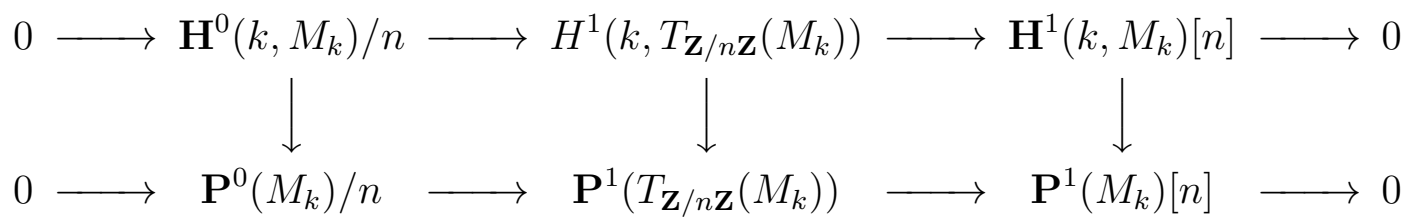

If we pass to the inverse limit over all $n$, the two lines remain left exact, whence a commutative exact diagram

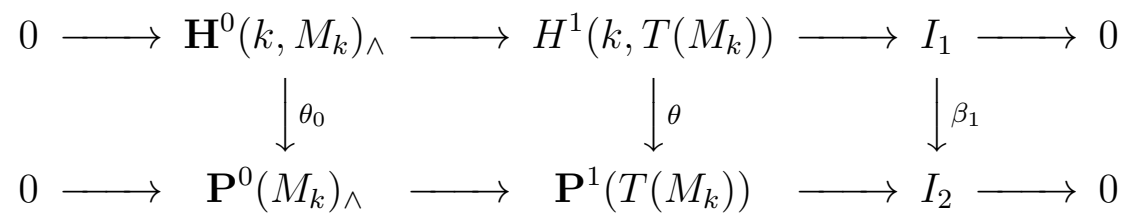

where we define $\mathbf{P}^{1}\left(T\left(M_{k}\right)\right)$ (resp. $H^{1}\left(k, T\left(M_{k}\right)\right)$ ) as the inverse limit of $\mathbf{P}^{1}\left(T_{\mathbf{Z} / n \mathbf{Z}}\left(M_{k}\right)\right)$ (resp. $\left.H^{1}\left(k, T_{\mathbf{Z} / n \mathbf{Z}}\left(M_{k}\right)\right)\right)$. Here $I_{1}, I_{2}$ are respectively subgroups of the full Tate modules $T\left(H^{1}\left(k, M_{k}\right)\right)$ and $T\left(\mathbf{P}^{1}\left(M_{k}\right)\right)$. In particular, Ker $\beta_{1}$ is a subgroup of $T\left(\amalg^{1}\left(M_{k}\right)\right)$ because the inverse limit functor is left exact. But $T\left(\amalg^{1}\left(M_{k}\right)\right)$ is zero thanks to the finiteness assumption on $\amalg^{1}\left(A_{k}\right)$ (which implies the finiteness of $\amalg^{1}\left(M_{k}\right)$ as in Corollary 4.9). 
Therefore we obtain that $\amalg_{\wedge}^{0}\left(M_{k}\right)=\operatorname{Ker} \theta_{0}$ is isomorphic to $\operatorname{Ker} \theta$. By Poitou-Tate duality for finite modules (18, VIII.6.8), there is a perfect pairing between the latter group and $\amalg^{2}\left(T\left(M_{k}^{*}\right)_{\text {tors }}\right)$. We conclude by observing that $\amalg^{2}\left(T\left(M_{k}^{*}\right)_{\text {tors }}\right)$ is also $\amalg^{2}\left(M_{k}^{*}\right)_{\text {tors }}$ by the same argument as in [16], I.6.8 (use the analogue of the exact sequence (11) for $M_{k}$ over Spec $k$ with $i=2$ and pass to the limit).

We now return to diagram (13) and prove:

Proposition 5.4 Keep the finiteness assumption on $\amalg^{1}\left(A_{k}\right)$. Then, with notations as in diagram (13), the natural map $\operatorname{ker} \theta_{0} \rightarrow \operatorname{ker} \beta_{0}$ is an isomorphism.

By virtue of the proposition, we may employ the notation $\amalg_{\wedge}^{0}\left(M_{k}\right)$ for ker $\beta_{0}$ as well and use the resulting duality. The finiteness assumption on $\amalg^{1}\left(A_{k}\right)$ is presumably superfluous here but we did not succeed in removing it (and use it elsewhere anyway).

For the proof we need a lemma about abelian groups.

Lemma 5.5 Let $A$ be a discrete abelian group of finite exponent $n$. Then the intersection of the finite index subroups of $A$ is trivial.

Proof : Consider the profinite group $A^{D}=\operatorname{Hom}(A, \mathbf{Z} / n)$. As $A^{D D}=A$, the statement is equivalent to saying that any character of $A^{D}$ vanishing on all finite subgroups of $A^{D}$ is trivial. This holds because all finitely generated subgroups of $A^{D}$ are finite.

Proof of Proposition 5.4: We begin by showing that the vertical maps in diagram (13) above are injective, whence the injectivity of the map ker $\theta_{0} \rightarrow$ ker $\beta_{0}$. For the left one, note that the topology on $\mathbf{H}^{0}(k, M)$ being discrete, injectivity means that any element of $\mathbf{H}^{0}(k, M)$ which is nontrivial modulo $n$ for some $n$ gives a nonzero element in some finite quotient - this holds by the lemma above.

For injectivity of the map $\mathbf{P}^{0}(k, M)_{\wedge} \rightarrow \mathbf{P}^{0}(k, M)^{\wedge}$, take an element $x=\left(x_{v}\right) \in \mathbf{P}^{0}\left(M_{k}\right)$ not lying in $n \mathbf{P}^{0}\left(M_{k}\right)$. We have to find an open subgroup of finite index avoiding $x$. By Lemma 5.3, there is a local component $x_{v}$ not lying in $n \mathbf{H}^{0}\left(\hat{k}_{v}, M\right)$. We thus get that for some $v$, our $x$ is not contained in the inverse image in $\mathbf{P}^{0}\left(M_{k}\right)$ of the subgroup $n \mathbf{H}^{0}\left(\hat{k}_{v}, M\right) \subset \mathbf{H}^{0}\left(\hat{k}_{v}, M\right)$, which is open of finite index, by definition of the topology on $\mathbf{H}^{0}\left(\hat{k}_{v}, M\right)$.

For the surjectivity of the map $\operatorname{ker} \theta_{0} \rightarrow \operatorname{ker} \beta_{0}$, remark first that ker $\theta_{0}$ is a profinite group, being dual to the torsion group $\amalg^{2}\left(M_{k}\right)$ by the previous proposition. Therefore $\left(\operatorname{ker} \theta_{0}\right)^{\wedge}=\operatorname{ker} \theta_{0}$, so by completing the exact 
sequence

$$
0 \rightarrow \operatorname{ker} \theta_{0} \rightarrow \mathbf{H}^{0}\left(k, M_{k}\right)_{\wedge} \rightarrow \operatorname{Im} \theta_{0} \rightarrow 0
$$

we get an exact sequence $\operatorname{ker} \theta_{0} \rightarrow \mathbf{H}^{0}\left(k, M_{k}\right)^{\wedge} \rightarrow\left(\operatorname{Im} \theta_{0}\right)^{\wedge} \rightarrow 0$. To conclude, it is thus enough to show the injectivity of the natural map $\left(\operatorname{Im} \theta_{0}\right)^{\wedge} \rightarrow$ $\mathbf{P}^{0}\left(M_{k}\right)^{\wedge}$. By the above considerations, $\mathbf{P}^{0}\left(M_{k}\right)_{\wedge}$ injects into its completion $\mathbf{P}^{0}\left(M_{k}\right)^{\wedge}$, so the completion of the subgroup $\operatorname{Im} \theta_{0}$ is simply its closure in $\mathbf{P}^{0}\left(M_{k}\right)^{\wedge}$, whence the claim. But there is a subtle point here: in this argument, $\operatorname{Im} \theta_{0}$ is equipped with the subspace topology inherited from $\mathbf{P}^{0}\left(M_{k}\right)_{\wedge}$, whereas in exact sequence (15) it carried the quotient topology from $\mathbf{H}^{0}\left(M_{k}\right)_{\wedge}$; we have to check that the two topologies are the same. It is enough to show that $\operatorname{Im} \theta_{0}$ is closed in $\mathbf{P}^{0}\left(M_{k}\right)_{\wedge}$, for then it is locally compact in the subspace topology and [8, 5.29 applies. To do so, observe that the image of the map $\theta$ in diagram (14) above equals the kernel of the map $\mathbf{P}^{1}\left(T\left(M_{k}\right)\right) \rightarrow H^{1}\left(k, T\left(M_{k}^{*}\right)_{\text {tors }}\right)^{D}$ coming from local duality, as a consequence of the Poitou-Tate sequence for finite modules ([18], VIII.6.13). Now a diagram chase in (14) using the injectivity of $\beta_{1}$ reveals that $\operatorname{Im} \theta_{0}$ is the kernel of the (continuous) composite map $\mathbf{P}^{0}\left(M_{k}\right)_{\wedge} \rightarrow \mathbf{P}^{1}\left(T\left(M_{k}\right)\right) \rightarrow$ $H^{1}\left(k, T\left(M_{k}^{*}\right)_{\text {tors }}\right)^{D}$, hence it is closed indeed.

We can now state the main result of this section.

Theorem 5.6 (Poitou-Tate exact sequence) Let $M_{k}$ be a 1-motive over $k$. Assume that $\amalg^{1}\left(A_{k}\right)$ and $\amalg^{1}\left(A_{k}^{*}\right)$ are finite, where $A_{k}$ is the abelian variety corresponding to $M_{k}$. Then there is a twelve term exact sequence of topological groups

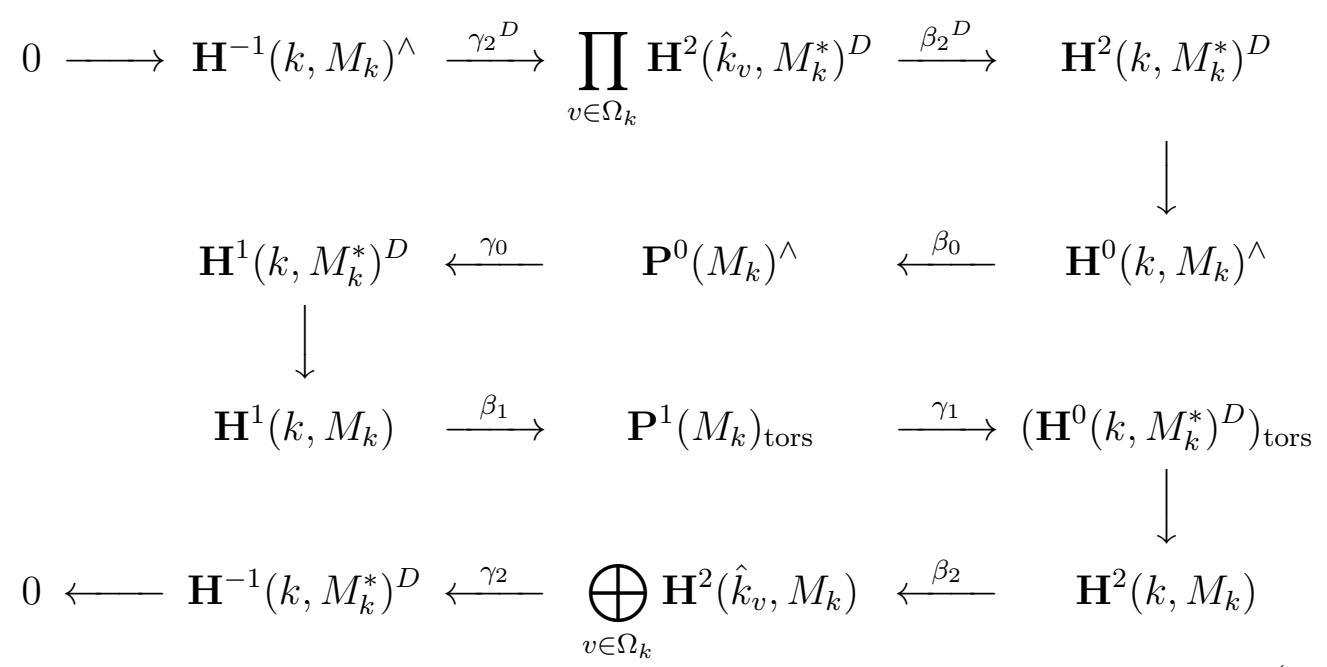


where the maps $\beta_{i}$ are the restriction maps defined at the beginning of this section, the maps $\gamma_{i}$ are induced by the local duality theorem of Section 2, and the unnamed maps come from the global duality results of Proposition 5.1 (completed by Proposition 5.4) and Corollary 4.9.

\section{Remarks 5.7}

1. In the above sequence the group $\mathbf{P}^{1}\left(M_{k}\right)_{\text {tors }}$ is equipped with the discrete topology, and not the subspace topology from $\mathbf{P}^{1}\left(M_{k}\right)$.

2. The sequence (16) is completely symmetric in the sense that if we replace $M_{k}$ by $M_{k}^{*}$ and dualise, we obtain exactly the same sequence.

3. If we only assume the finiteness of $\amalg^{1}\left(A_{k}\right)\{\ell\}$ and $\amalg^{1}\left(A_{k}^{*}\right)\{\ell\}$ for some prime number $\ell$, then the analogue of the exact sequence (16) still holds, with profinite completions replaced by $\ell$-adic completions and the torsion groups involved by their $\ell$-primary part.

4. Some special cases of the theorem are worth noting. For $M_{k}=\left[0 \rightarrow A_{k}\right]$ we get the ten-term exact sequence of [16], I.6.14. For $M_{k}=[0 \rightarrow$ $T_{k}$ ] one can show (see proof of Proposition 5.9 below) that $\beta_{2}^{D}$ is an isomorphism, hence we obtain a classical (?) nine-term exact sequence; the case $M_{k}=Y_{k}[1]$ is symmetric (compare [16], I.4.20).

The proof will use the following lemma.

Lemma 5.8 Let $M_{k}$ be a 1-motive over $k$. Then $\mathbf{H}^{0}\left(k, M_{k}\right)_{\text {tors }}$ is finite.

Proof : $\quad$ Let $M_{k}^{\prime}=M_{k} / W_{-2}\left(M_{k}\right)=\left[Y_{k} \rightarrow A_{k}\right]$. From the exact sequence

$$
H^{0}\left(k, Y_{k}\right) \rightarrow H^{0}\left(k, A_{k}\right) \rightarrow \mathbf{H}^{0}\left(k, M_{k}^{\prime}\right) \rightarrow H^{1}\left(k, Y_{k}\right)
$$

we deduce that $\mathbf{H}^{0}\left(k, M_{k}^{\prime}\right)$ is of finite type because $H^{0}\left(k, A_{k}\right)$ is of finite type (Mordell-Weil theorem) and $H^{1}\left(k, Y_{k}\right)$ is finite (because $Y_{k}(\bar{k})$ is a lattice). There is also an exact sequence

$$
\mathbf{H}^{-1}\left(k, M_{k}^{\prime}\right) \rightarrow H^{0}\left(k, T_{k}\right) \rightarrow \mathbf{H}^{0}\left(k, M_{k}\right) \rightarrow \mathbf{H}^{0}\left(k, M_{k}^{\prime}\right)
$$

where $T_{k}$ is the torus corresponding to $M_{k}$. It is therefore sufficient to show that the torsion subgroup of the group $B:=T_{k}(k) / \operatorname{Im} \mathbf{H}^{-1}\left(k, M_{k}^{\prime}\right)$ is finite. Choose generators $a_{1}, \ldots, a_{r}$ of the lattice $\mathbf{H}^{-1}\left(k, M_{k}^{\prime}\right)$, and let $b_{1}, \ldots, b_{r}$ be their images in $T_{k}(k)$. We can find an open subset $U$ of $\operatorname{Spec} \mathcal{O}_{k}$ such that $T_{k}$ extends to a torus $T$ over $U$, and $b_{i} \in H^{0}(U, T)$ for any $i \in\{1, \ldots, r\}$. In 
this way, each $x \in B_{\text {tors }}$ is the image in $B$ of an element $y \in T_{k}(k)$ for which $y^{n} \in H^{0}(U, T)$ for some $n>0$. Let $V / U$ be an étale covering such that $T$ splits over $V$, i.e. it becomes isomorphic to some power $\mathbf{G}_{m}^{N}$. If $L$ denotes the fraction field of $V$, we have $T_{k}(L) / H^{0}(V, T) \cong\left(L^{\times} / H^{0}\left(V, \mathbf{G}_{m}\right)\right)^{N}$ which is naturally a subgroup of the free abelian group $\operatorname{Div}(V)^{N}$; in particular, it has no torsion. Therefore, since $y^{n} \in H^{0}(V, T)$ via the inclusion $H^{0}(U, T) \rightarrow$ $H^{0}(V, T)$ we get that $y \in H^{0}(V, T)=H^{0}\left(V, \mathbf{G}_{m}\right)^{N}$. Let $H$ be the subgroup of $H^{0}(V, T)$ generated by the $y$ 's; since $H^{0}\left(V, \mathbf{G}_{m}\right)$ is of finite type by Dirichlet's Unit Theorem, so is $H$. We thus get a surjection from the finitely generated group $H$ to the torsion group $B_{\text {tors }}$, whence the claim.

Proof of Theorem [5.6: The first line is dual to the last one, so for its exactness it is enough to show the exactness of the latter. We proceed as in [16], I.6.13 (b). For each $n>0$, we have an exact commutative diagram (using the exact sequence (111) and the Poitou-Tate sequence for the finite module $\left.T_{\mathbf{Z} / n \mathbf{Z}}\left(M_{k}\right)\right)$ :

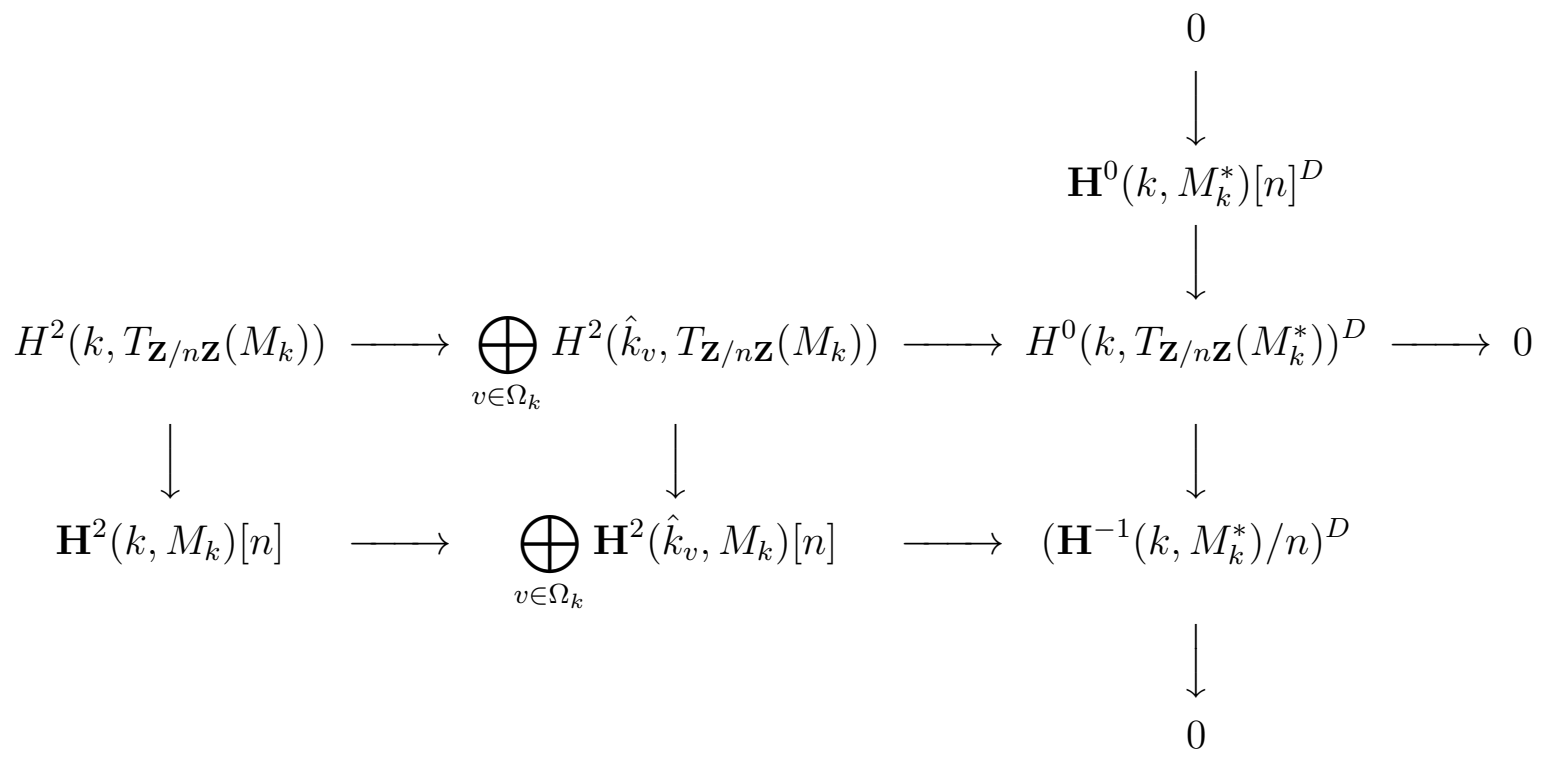

By Lemma 5.8, the full Tate module $T\left(\mathbf{H}^{0}\left(k, M_{k}^{*}\right)\right)$ is trivial. Therefore taking the inductive limit over all $n$, we obtain the exact commutative diagram, where the right vertical map is an isomorphism:

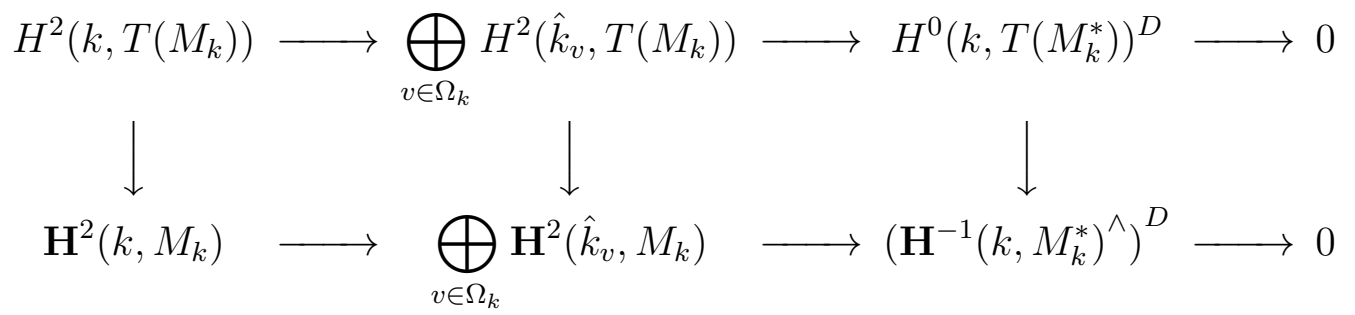


We remark that the first two vertical maps are also isomorphisms by the same argument as at the end of the proof of Proposition [5.1. Since the first row is exact by the Poitou-Tate sequence for finite modules ([18, VIII.6.13), so is the second one. Therefore the exactness of the last line of (16) (and hence that of the first) follows noting that the dual of the profinite completion of the lattice $\mathbf{H}^{-1}\left(k, M_{k}^{*}\right)$ is the same as the dual of $\mathbf{H}^{-1}\left(k, M_{k}^{*}\right)$ itself.

To prove exactness of the second line, note first that it is none but the profinite completion of the sequence

$$
H^{0}\left(k, M_{k}\right)_{\wedge} \stackrel{\theta_{0}}{\rightarrow} \mathbf{P}^{0}\left(M_{k}\right)_{\wedge} \stackrel{\gamma_{0}^{\prime}}{\rightarrow} H^{1}\left(k, M_{k}^{*}\right)^{D},
$$

where the map $\gamma_{0}^{\prime}$ is induced by local duality, taking Lemma 5.3 into account. First we show that this latter sequence is a complex. The map $\gamma_{0}^{\prime}$ has the following concrete description at a finite level: for $\alpha=\left(\alpha_{v}\right) \in \mathbf{P}^{0}\left(M_{k}\right) / n$ and $\beta \in \mathbf{H}^{1}\left(k, M_{k}^{*}\right)[n]$, denote by $\beta_{v}$ the image of $\beta$ in $\mathbf{H}^{1}\left(\hat{k}_{v}, M_{k}^{*}\right)[n]$. Then $\left[\gamma_{0}^{\prime}(\alpha)\right](\beta)$ is the sum over all $v$ of the elements $j_{v}\left(\alpha_{v} \cup \beta_{v}\right)$, where $\left(\alpha_{v} \cup \beta_{v}\right) \in$ $H^{2}\left(\hat{k}_{v}, \mu_{n}\right)$ via the pairing $M \times M^{*} \rightarrow \mathbf{G}_{m}[1]$, and $j_{v}$ is the local invariant. (The sum is finite by virtue of the property $H^{2}\left(\widehat{\mathcal{O}}_{v}, \mu_{n}\right)=0$ because the elements $\alpha_{v}$ and $\beta_{v}$ are unramified for almost all $v$.) Then $\gamma_{0}^{\prime} \circ \theta_{0}=0$ follows (after passing to the limit) from the reciprocity law of global class field theory, according to which the sequence

$$
0 \rightarrow H^{2}\left(k, \mu_{n}\right) \rightarrow \bigoplus_{v \in \Omega_{k}} H^{2}\left(\hat{k}_{v}, \mu_{n}\right) \stackrel{\sum j_{v}}{\rightarrow} \mathbf{Z} / n \rightarrow 0
$$

is a complex.

For the exactness of the sequence (17) recall that, as remarked at the end of the proof of Proposition 5.4. diagram (14) and the Poitou-Tate sequence for finite modules imply that $\operatorname{Im} \theta_{0}$ is the kernel of the composed map

$$
\mathbf{P}^{0}\left(M_{k}\right)_{\wedge} \rightarrow \mathbf{P}^{1}\left(\left(T\left(M_{k}\right)\right) \rightarrow H^{1}\left(k, T\left(M_{k}^{*}\right)_{\text {tors }}\right)^{D} .\right.
$$

The claimed exactness then follows from the commutative diagram

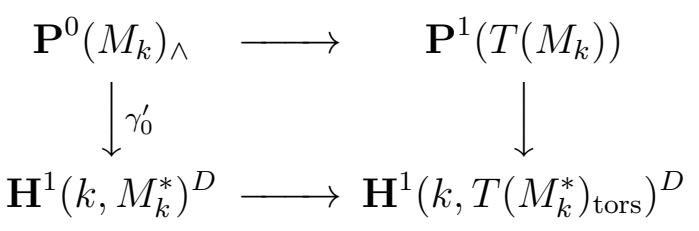

whose commutativity arises from the compatibility of the duality pairings for 1-motives and their " $n$-adic" realisations via the Kummer map.

Now we show that the profinite completion of sequence (17), i.e. the second row of diagram (16) remains exact. This follows by an argument 
similar to the one at the end of the proof of Proposition [5.4, once having checked that $\operatorname{Im} \gamma_{0}^{\prime}$ is closed in $\mathbf{H}^{1}\left(k, M_{k}^{*}\right)^{D}$ and $\left(\operatorname{Im} \gamma_{0}^{\prime}\right)^{\wedge}=\operatorname{Im} \gamma_{0}^{\prime}$. To see this, note that by applying the snake lemma to diagram (14) we get that Coker $\theta_{0}$ (with the quotient topology) injects as a closed subgroup into Coker $\theta$. But one sees using the Poitou-Tate sequence for finite modules that the latter group is profinite, hence so is $\operatorname{Im} \gamma_{0}^{\prime}$; in particular, it is compact and hence closed in $\mathbf{H}^{1}\left(k, M_{k}^{*}\right)^{D}$.

Next, remark that by definition of the restricted product topology and Theorem 2.10, the dual of the group $\mathbf{P}^{0}\left(M_{k}\right)$ (equipped with the restricted product topology) is $\mathbf{P}^{1}\left(M_{k}^{*}\right)$. Thus the dual of $\mathbf{P}^{0}\left(M_{k}\right)^{\wedge}$ is $\mathbf{P}^{1}\left(M_{k}^{*}\right)_{\text {tors }}$. Therefore we obtain the third line by dualising the second one (and exchanging the roles of $M$ and $M^{*}$ ), which consists of profinite groups.

Finally, the exactness of the sequence (16) at the "corners" follows immediately, in the first two rows, from the dualities $\amalg_{\wedge}^{0}\left(M_{k}\right) \cong \amalg^{2}\left(M_{k}^{*}\right)^{D}$ (Proposition 5.1 combined with Proposition 5.4) and $\amalg^{1}\left(M_{k}\right) \cong \amalg^{1}\left(M_{k}^{*}\right)^{D}$ (Corollary 4.9), respectively; the remaining corner is dual to the first one.

We conclude with the following complement.

Proposition 5.9 Let $M_{k}$ be a 1-motive over $k$. Then the natural map $\mathbf{H}^{i}\left(k, M_{k}\right) \rightarrow \bigoplus_{v \in \Omega_{\infty}} \mathbf{H}^{i}\left(k_{v}, M_{k}\right)$ is an isomorphism for $i \geq 3$.

Proof : When $M=[0 \rightarrow G]$, this follows immediately by devissage from [16], I.4.21, and I.6.13 (c). To deal with the general case, it is sufficient to show that the map $f_{i}: H^{i}\left(k, Y_{k}\right) \rightarrow \bigoplus_{v \in \Omega_{\mathbf{R}}} H^{i}\left(k_{v}, Y_{k}\right)$ is an isomorphism for $i \geq 3$. Using the exact sequence

$$
0 \rightarrow H^{i}\left(k, Y_{k}\right) / n \rightarrow H^{i}\left(k, Y_{k} / n Y_{k}\right) \rightarrow H^{i+1}\left(k, Y_{k}\right)[n] \rightarrow 0
$$

for each $n>0$, we reduce to the case $i=3$. The last line of the PoitouTate exact sequence for $M_{k}=Y_{k}[1]$ yields the surjectivity of $f_{3}$ because $\hat{k}_{v}$ is of strict cohomological dimension 2 for $v$ finite. On the other hand, we remark that $H^{3}(k, \mathbf{Z})=0\left([16]\right.$, I.4.17), hence $H^{3}\left(k, Y_{k}\right)=H^{3}\left(k, Y_{k}\right)[n]$ for some $n>0$ by a restriction-corestriction argument. In particular the divisible subgroup of $H^{3}\left(k, Y_{k}\right)$ is zero. The injectivity of $f_{3}$ now follows from Proposition 4.12 applied to $M_{k}=T_{k}^{*}$, where $T_{k}^{*}$ is the torus with module of characters $Y_{k}$.

Remark 5.10 The global results in this paper still hold (with the same proofs) if we replace the number field $k$ by a function field in one variable 
over a finite field, provided that one ignores the $p$-primary torsion part of the groups under consideration, where $p=$ char $k$ ). We leave the verification of this to the readers.

\section{Comparison with the Cassels-Tate pairing}

In this section, we give a definition of the pairing of Theorem 0.2 purely in terms of Galois cohomology and show that in the case $M=[0 \rightarrow A]$ it reduces to the classical Cassels-Tate pairing for abelian varieties.

The idea is to use the diminished cup-product construction discovered by Poonen and Stoll (see [20], pp. 1117-1118). One could present it in a general categorical setting but for the ease of exposition we stick to the special situation we have. So assume given three exact sequences

$$
\begin{gathered}
0 \rightarrow M_{1} \rightarrow M_{2} \rightarrow M_{3} \rightarrow 0 \\
0 \rightarrow N_{1} \rightarrow N_{2} \rightarrow N_{3} \rightarrow 0 \\
0 \rightarrow P_{1} \rightarrow P_{2} \rightarrow P_{3} \rightarrow 0
\end{gathered}
$$

where the $M_{i}, N_{i}, P_{i}$ are complexes of abelian étale sheaves over some scheme $S$. Assume further given a pairing $M_{2} \otimes^{\mathbf{L}} N_{2} \rightarrow P_{2}$ in the derived category that maps $M_{1} \otimes^{\mathbf{L}} N_{1}$ to $P_{1}$. Then one has a pairing

$\operatorname{Ker}\left[\mathbf{H}^{i}\left(S, M_{1}\right) \rightarrow \mathbf{H}^{i}\left(S, M_{2}\right)\right] \times \operatorname{Ker}\left[\mathbf{H}^{j}\left(S, N_{1}\right) \rightarrow \mathbf{H}^{j}\left(S, N_{2}\right)\right] \rightarrow \mathbf{H}^{i+j-1}\left(S, P_{3}\right)$

defined as follows. Any element $a$ of $\operatorname{ker}\left[\mathbf{H}^{i}\left(S, M_{1}\right) \rightarrow \mathbf{H}^{i}\left(S, M_{2}\right)\right]$ comes from an element $b$ of $\mathbf{H}^{i-1}\left(S, M_{3}\right)$. Since the above pairing induces a pairing $M_{3} \otimes^{\mathbf{L}} N_{1} \rightarrow P_{3}$, our $b$ can be cupped with $a^{\prime} \in \operatorname{ker}\left[\mathbf{H}^{j}\left(S, N_{1}\right) \rightarrow \mathbf{H}^{j}\left(S, N_{2}\right)\right]$ to get an element in $\mathbf{H}^{i+j-1}\left(S, P_{3}\right)$. It follows from the fact that $a^{\prime}$ maps to 0 in $\mathbf{H}^{j}\left(S, N_{2}\right)$ that this definition does not depend on the choice of $b$.

Now apply this in the following situation. Let $\mathbf{A}_{k}$ be the adèle ring of the number field $k, S=$ Spec $k$ and $M_{k}$ a 1-motive over $k$. Consider étale sheaves over Spec $k$ as Gal $(k)$-modules and put $M_{1}=M_{k}(\bar{k}), M_{2}=M_{k}\left(\bar{k} \otimes_{k} \mathbf{A}_{k}\right)$, $N_{1}=M_{k}^{*}(\bar{k}), N_{2}=M_{k}^{*}\left(\bar{k} \otimes_{k} \mathbf{A}_{k}\right), P_{1}=\mathbf{G}_{m}(\bar{k})[1], P_{2}=\mathbf{G}_{m}\left(\bar{k} \otimes_{k} \mathbf{A}_{k}\right)[1]$ and define $M_{3}, N_{3}, P_{3}$ to make the above sequences exact. The pairing $M_{k} \otimes^{\mathbf{L}} M_{k}^{*} \rightarrow \mathbf{G}_{m}[1]$ induces the pairing $M_{2} \otimes^{\mathbf{L}} N_{2} \rightarrow P_{2}$ required in the above situation. Note that we have

$$
\begin{aligned}
& \amalg^{i}\left(M_{k}\right)=\operatorname{Ker}\left[\mathbf{H}^{i}\left(k, M_{1}\right) \rightarrow \mathbf{H}^{i}\left(k, M_{2}\right)\right], \\
& \amalg^{j}\left(M_{k}^{*}\right)=\operatorname{Ker}\left[\mathbf{H}^{j}\left(k, N_{1}\right) \rightarrow \mathbf{H}^{j}\left(k, N_{2}\right)\right] .
\end{aligned}
$$


Finally, class field theory tells us that the cokernel of the map $H^{2}\left(k, \mathbf{G}_{m}\right) \rightarrow$ $H^{2}\left(k, \mathbf{G}_{m}\left(\bar{k} \otimes_{k} \mathbf{A}_{k}\right)\right)$ is isomorphic to $\mathbf{Q} / \mathbf{Z}$; indeed, one has

$$
H^{2}\left(k, \mathbf{G}_{m}\left(\bar{k} \otimes_{k} \mathbf{A}_{k}\right)\right)=\bigoplus_{v} H^{2}\left(k_{v}, \mathbf{G}_{m}\right)
$$

by Shapiro's lemma (and the isomorphisms $H^{2}\left(k_{v}, \mathbf{G}_{m}\right) \cong H^{2}\left(\hat{k}_{v}, \mathbf{G}_{m}\right)$, cf. Lemma 2.77), so the claim follows from the exactness of the sequence (18) (18), Theorem 8.1.17) in view of the vanishing of $H^{3}\left(k, \mathbf{G}_{m}\right)([18,8.3 .10$ (iv)).

Putting all this together, we get that the diminished cup-product construction yields for $j=2-i$ pairings

$$
\amalg^{i}\left(M_{k}\right) \times \amalg^{2-i}\left(M_{k}\right) \rightarrow \mathbf{Q} / \mathbf{Z}
$$

for $i=0,1$.

Proposition 6.1 The above pairings coincide with those constructed in Section 4 .

Proof : $\quad$ Assume $M_{k}$ extends to a 1-motive $M$ over an open subset $U \subset$ Spec $k$; let $\Sigma$ denote the finite set of places of $k$ which are real or coming from closed points of $\operatorname{Spec} \mathcal{O}_{k}$ outside $U$. Apply the diminished cup-product construction for $S=U, M_{1}=M, M_{2}=p_{*} p^{*} M, N_{1}=M^{*}, N_{2}=p_{*} p^{*} M^{*}$, $P_{1}=\mathbf{G}_{m}[1], P_{2}=p_{*} p^{*} \mathbf{G}_{m}[1]$, where $p: \oplus_{v \in \Sigma} \operatorname{Spec} k_{v} \rightarrow U$ is the canonical morphism (here we use the conventions of Section 3 for $k_{v}$ ). Note that, in the notation of Section 3,

$$
D^{i}(U, M)=\operatorname{ker}\left[\mathbf{H}^{i}\left(U, M_{1}\right) \rightarrow \mathbf{H}^{i}\left(U, M_{2}\right)\right]
$$

and

$$
D^{j}\left(U, M^{*}\right)=\operatorname{ker}\left[\mathbf{H}^{j}\left(U, N_{1}\right) \rightarrow \mathbf{H}^{j}\left(U, N_{2}\right)\right] .
$$

Moreover, one has

$$
\operatorname{coker}\left[H^{2}\left(U, \mathbf{G}_{m}\right) \rightarrow H^{2}\left(U, p_{*} p^{*} \mathbf{G}_{m}\right)\right] \cong H_{c}^{3}\left(U, \mathbf{G}_{m}\right) \cong \mathbf{Q} / \mathbf{Z}
$$

where the first isomorphism comes from Shapiro's lemma in the étale setting and the second from class field theory, both combined with the fundamental long exact sequence of compact support cohomology (the second isomorphism was already used to construct the pairings of Chapter 3). For $i=0,1$, $j=2-i$ one thus gets pairings

$$
D^{i}(U, M) \times D^{2-i}(U, M) \rightarrow \mathbf{Q} / \mathbf{Z}
$$


which are manifestly the same as those of Corollary 3.5. Moreover, over a sufficiently small $U$ these are by construction compatible with the above definition of the pairing (19).

Proposition 6.2 For $M_{k}=\left[0 \rightarrow A_{k}\right]$ the above pairing reduces to the classical Cassels-Tate pairing.

Proof : This was basically proven in [20]. There one finds four equivalent definitions of the Cassels-Tate pairing, two of them using the diminished cupproduct construction for explicitly defined pairings $A_{k}(\bar{k}) \times A_{k}^{*}(\bar{k}) \rightarrow \mathbf{G}_{m}[1]$. For instance, one of them (called the Albanese-Picard pairing by [20]) is given as follows: replace $A_{k}(\bar{k})$ with the quasi-isomorphic complex $C_{1}=[Y(A) \rightarrow$ $Z(A)$, where $Z(A)$ is the group of zero-cycles on $A_{\bar{k}}=A_{k} \times_{k} \bar{k}$ and $Y(A)$ is the kernel of the natural summation map $Z(A) \rightarrow A(\bar{k})$, and replace $A_{k}^{*}(\bar{k})$ by the complex $C_{2}=\left[\bar{k}\left(A_{\bar{k}}\right)^{\times} / \bar{k}^{\times} \rightarrow \operatorname{Div}^{0}\left(A_{\bar{k}}\right)\right]$, where $\operatorname{Div}^{0}$ stands for divisors algebraically equivalent to 0 . Now define a map $C_{1} \otimes C_{2} \rightarrow \mathbf{G}_{m}(\bar{k})[1]$ using the partially defined pairings

$$
Z(A) \times\left[\bar{k}\left(A_{\bar{k}}\right)^{\times} / \bar{k}^{\times}\right] \rightarrow \bar{k}^{\times}
$$

and

$$
\operatorname{Div}^{0}\left(A_{\bar{k}}\right) \times Y(A) \rightarrow \bar{k}^{\times}
$$

where the first is defined on $([20$, p. 1116) using the Poincaré divisor on $A \times A^{*}$ and the second by evaluation. The resulting pairing $A_{k}(\bar{k}) \times A_{k}^{*}(\bar{k}) \rightarrow$ $\mathbf{G}_{m}[1]$ is one of the classical definitions of the duality pairing for abelian varieties over an algebraically closed field.

Remark 6.3 Similarly, one verifies that in the case $M_{k}=\left[0 \rightarrow T_{k}\right]$ one gets the usual pairing between the Tate-Shafarevich groups of the torus $T_{k}$ and its character module.

Remark 6.4 In [5], Flach defines a Cassels-Tate pairing for finite dimensional continuous $\ell$-adic representations of the Galois group of a number field $k$ that stabilize some lattice. Comparing the above construction with his shows that in the case when the representation comes from the $\ell$-adic realisation of a 1-motive $M_{k}$ the two pairings are compatible. In the special cases $M_{k}=[\mathbf{Z} \rightarrow 0]$ and $M_{k}=\left[0 \rightarrow A_{k}\right]$ this is already pointed out in Flach's paper (see also [6] for the latter case). So our result can be interpreted as a "motivic version" of Flach's pairing for motives of dimension one. 


\section{Appendix : Completion of topological abelian groups}

In this appendix we collect some (probably well-known) results that we needed in the paper.

Proposition Let $0 \rightarrow A \stackrel{i}{\rightarrow} B \stackrel{p}{\rightarrow} C \rightarrow 0$ be an exact sequence in the category of topological abelian groups.

1. If $i$ is strict with open image, then the map $B^{D} \rightarrow A^{D}$ is surjective.

2. Assume that the map $p: B \rightarrow C$ is open. Then the sequences

$$
\begin{gathered}
A^{\wedge} \rightarrow B^{\wedge} \rightarrow C^{\wedge} \rightarrow 0 \\
0 \rightarrow C^{D} \rightarrow B^{D} \rightarrow A^{D}
\end{gathered}
$$

are exact.

3. Assume that $p$ is open and $i$ is strict with closed image. Suppose further that $B$ is Hausdorff, locally compact, completely disconnected, and compactly generated. Then the sequences

$$
\begin{aligned}
& 0 \rightarrow A^{\wedge} \rightarrow B^{\wedge} \rightarrow C^{\wedge} \rightarrow 0 \\
& 0 \rightarrow C^{D} \rightarrow B^{D} \rightarrow A^{D} \rightarrow 0
\end{aligned}
$$

are exact, the map $B \rightarrow B^{\wedge}$ is injective, and $\left(B^{\wedge}\right)^{D}=B^{D}$.

Proof : 1. If $i$ is strict and $i(A)$ is open in $B$, then any continuous homomorphism $A \rightarrow \mathbf{Q} / \mathbf{Z}$ induces a continuous homomorphism $s: i(A) \rightarrow$ $\mathbf{Q} / \mathbf{Z}$ which in particular has open kernel. By divisibility of $\mathbf{Q} / \mathbf{Z}$ this extends to a homomorphism $\bar{s}: B \rightarrow \mathbf{Q} / \mathbf{Z}$. Since $i(A)$ is open in $B, \operatorname{Ker} \bar{s}$ is also open in $B$ being a union of cosets of Ker $s$, whence the continuity of $\bar{s}$.

2. To get the first exact sequence, the only nontrivial point consists of proving that an element $b \in B^{\wedge}$ whose image in $C^{\wedge}$ is trivial comes from $A^{\wedge}$. Let $B^{\prime} \subset B$ be an open subgroup of finite index. Then $p\left(B^{\prime}\right) \subset C$ is of finite index and is open because $p$ is open. Since $i$ is continuous, $\left(A \cap B^{\prime}\right) \subset A$ is open and of finite index as well. Now we use the exact sequence

$$
0 \rightarrow A /\left(A \cap B^{\prime}\right) \rightarrow B / B^{\prime} \rightarrow C / p\left(B^{\prime}\right) \rightarrow 0
$$

where $B^{\prime}$ runs over the finite index open subgroups of $B$.

The second exact sequence follows immediately from the fact that the group $C \simeq B / i(A)$ is equipped with the quotient topology ( $p$ being an open map). 
3. Let us show the left exactness of the first sequence. Since $i$ is strict with closed image, we can assume that $A$ is a closed subgroup of $B$ with the induced topology. We have to show that if $A^{\prime} \subset A$ is open and of finite index, then there exists an open subgroup of finite index $B^{\prime} \subset B$ such that $B^{\prime} \cap A \subset A^{\prime}$. Replacing $A$ and $B$ by $A / A^{\prime}$ and $B / A^{\prime}$ (equipped with the quotient topology), we reduce to the case when $A$ is a finite subgroup and we must show that $B$ contains a finite index open subgroup $B^{\prime}$ with $B^{\prime} \cap A=\{0\}$. To do so, it is sufficient (using the finiteness of $A$ ) to prove that the intersection of all finite index open subgroups of $B$ is zero, that is $B \hookrightarrow B^{\wedge}$. By [8, II.9.8. (in the special case when $B$ is completely disconnected), $B$ is topologically isomorphic to a product $\mathbf{Z}^{b} \times K$ with $K$ compact (hence profinite), thus $B \hookrightarrow B^{\wedge}=\widehat{Z}^{b} \times K$.

This also shows that $\left(B^{\wedge}\right)^{D}=B^{D}$. The projection $L$ of $A$ on $\mathbf{Z}^{b} \subset B$ is a discrete lattice and $A$ is topologically isomorphic to $L \times(A \cap K)$, hence $\left(A^{\wedge}\right)^{D}=A^{D}$. This proves the exactness of the second sequence.

\section{References}

[1] M. Artin, A. Grothendieck, J.-L. Verdier (eds.), Théorie des topos et cohomologie étale des schémas (SGA 4), Lecture Notes in Math. 269, Springer-Verlag 1972.

[2] V. G. Berkovich, Duality theorems in Galois cohomology of commutative algebraic groups, Selecta Math. Soviet. 6 (1987), 201-296.

[3] P. Deligne, Théorie de Hodge III, Inst. Hautes Études Sci. Publ. Math. 44 (1974), 5-77.

[4] C. Deninger, An extension of Artin-Verdier duality to non-torsion sheaves, J. reine angew. Math. 366 (1986), 18-31.

[5] M. Flach, A generalisation of the Cassels-Tate pairing, J. reine angew. Math. 412 (1990), 113-127.

[6] J.-M. Fontaine, B. Perrin-Riou, Autour des conjectures de Bloch-Kato: cohomologie galoisienne et valeurs de fonctions L, in U. Jannsen et al. (eds.) Motives, Proc. Symp. Pure Math. 55, vol. 1., pp. 599-706.

[7] M. J. Greenberg, Rational points in Henselian discrete valuation rings, Inst. Hautes Études Sci. Publ. Math. 31 (1966), 59-64.

[8] E. Hewitt, K. Ross, Abstract harmonic analysis I, Springer-Verlag 1963. 
[9] K. Kato, A Hasse principle for two dimensional global fields, J. reine angew. Math. 366 (1986), 142-183.

[10] R. Kottwitz, Stable trace formula: cuspidal tempered terms. Duke Math. J. 51 (1984), 611-650.

[11] R. Kottwitz, D. Shelstad, Foundations of twisted endoscopy, Astérisque 255, 1999.

[12] S. Lang, Algebraic groups over finite fields, Amer. J. Math. 78 (1956), $555-563$.

[13] G. A. Margulis, Discrete subgroups of semisimple Lie groups, Ergebnisse der Mathematik und ihrer Grenzgebiete (3) 17, Springer-Verlag, Berlin, 1991.

[14] B. Mazur, Notes on étale cohomology of number fields, Ann. Sci. Éc. Norm. Sup. 6 (1973), 521-556.

[15] J.S. Milne, Étale Cohomology, Princeton University Press, 1980.

[16] J.S. Milne, Arithmetic Duality Theorems, Academic Press, 1986.

[17] M. Nagata, Theory of commutative fields, Translations of Mathematical Monographs, vol. 125, American Mathematical Society, 1993.

[18] J. Neukirch, A. Schmidt, K. Wingberg : Cohomology of number fields, Grundlehren der Math. Wiss. 323, Springer-Verlag, 2000.

[19] F. Oort, Commutative group schemes, Lecture Notes in Math. 15, Springer-Verlag 1966.

[20] B. Poonen, M. Stoll, The Cassels-Tate pairing on polarized abelian varieties, Ann. of Math. 150 (1999), 1109-1149.

[21] N. Ramachandran, Duality of Albanese and Picard 1-motives, K-theory 21 (2001), 271-301.

[22] M. Raynaud, 1-motifs et monodromie géométrique, in Périodes $p$ adiques, Astérisque 223 (1994), 295-319.

[23] J-P. Serre, Cohomologie Galoisienne (cinquième édition, révisée et complétée), Lecture Notes in Math. 5, Springer Verlag, 1994.

[24] J-P. Serre, Morphismes universels et variété d'Albanese, Séminaire Chevalley, année 1958/58, exposé 10. 
[25] J-P. Serre, Morphismes universels et différentielles de troisième espèce, Séminaire Chevalley, année 1958/59, exposé 11.

[26] A. N. Skorobogatov, Torsors and rational points, Cambridge University Press, 2001.

[27] J. Tate, The cohomology groups of tori in finite Galois extensions of number fields, Nagoya Math. J. 27 (1966), 709-719.

[28] J. Tate, WC-groups over p-adic fields, Séminaire Bourbaki, année $1957 / 58$, exposé 156.

[29] J. Tate, Duality theorems in Galois cohomology over number fields, Proc. Internat. Congr. Mathematicians (Stockholm, 1962), 288-295.

[30] Th. Zink, Appendix 2 in K. Haberland, Galois cohomology of number fields, VEB Deutscher Verlag der Wissenschaften, Berlin, 1978.

David Harari

DMA

Ecole Normale Supérieure

45, rue d'Ulm

F-75230 Paris Cedex 05

France

David.Harari@ens.fr
Tamás Szamuely

Alfréd Rényi Institute of Mathematics

Hungarian Academy of Sciences

PO Box 127

H-1364 Budapest

Hungary

szamuely@renyi.hu 